\title{
Paysages et territoires du patrimoine industriel au Royaume-Uni
}

Landscapes and territories of industrial heritage in United-Kingdom

Landschaften und Räume des industriellen Erbes in dem Vereinigten Königreich

\section{Simon Edelblutte}

\section{(2) OpenEdition}

Journals

\section{Édition électronique}

URL : http://journals.openedition.org/rge/1165

DOI : $10.4000 /$ rge. 1165

ISSN : $2108-6478$

Éditeur

Association des géographes de l'Est

Édition imprimée

Date de publication : 1 janvier 2008

ISSN : 0035-3213

\section{Référence électronique}

Simon Edelblutte, «Paysages et territoires du patrimoine industriel au Royaume-Uni », Revue

Géographique de l'Est [En ligne], vol. 48 / 1-2 | 2008, mis en ligne le 08 octobre 2011, consulté le 08 septembre 2020. URL : http://journals.openedition.org/rge/1165; DOI : https://doi.org/10.4000/rge. 1165

Ce document a été généré automatiquement le 8 septembre 2020

Tous droits réservés 


\title{
Paysages et territoires du patrimoine industriel au Royaume- Uni
}

Landscapes and territories of industrial heritage in United-Kingdom Landschaften und Räume des industriellen Erbes in dem Vereinigten Königreich

\author{
Simon Edelblutte
}

L'auteur remercie les deux relecteurs pour les très utiles conseils bibliographiques

\section{Introduction}

1 La recomposition des territoires, leur reconversion et, en particulier, le renouveau urbain sont des notions largement développées depuis plus de 20 ans, notamment en géographie urbaine (Chaline, 1999; Burgel, 2001; Veschambre, 2005). Dans ce mouvement, la place du patrimoine est croissante. En effet, négligé dans les années de rénovation urbaine brutale (années 1950 à 1970), il s'est imposé assez rapidement dans les cœurs urbains tant il est devenu évident que les éléments du passé, au delà de leur intérêt intrinsèque, sont aussi des éléments essentiels de l'identité des habitants d'un lieu et, à ce titre, ne peuvent plus être balayés brutalement.

2 Cette notion de patrimoine, au départ restreinte à des «biens qui descendent, suivant la loi, des pères et des mères à leurs enfants» (Choay, Merlin, 2005), a été peu à peu élargie pour devenir " un ensemble de représentations, d'attributs fixés sur un objet non contemporain (cuuvre, bâtiment, paysage, site...) dont est décrétée collectivement l'importance intrinsèque qui exige qu'on le conserve» (Lévy, Lussault, 2004). C'est cet élargissement de la notion de patrimoine, de l'objet au site et jusqu'au paysage et au territoire, objets éminemment géographiques, qui a donné sa place au géographe dans ces travaux. Aux côtés des archéologues, des historiens, des sociologues, etc., il peut ainsi intégrer la notion de patrimoine dans l'étude des territoires et de leur recomposition. Les objets industriels, puis les bâtiments et, au delà, les sites et les paysages, entrent dans cette définition du 
patrimoine à condition qu'on leur reconnaisse un intérêt particulier pour la société, ce qui est vérifié depuis peu dans le cas de l'industrie. En effet, dans ce domaine, le ressenti des populations est souvent négatif et la notion de patrimoine s'est imposée plus tardivement que dans d'autres domaines (Edelblutte, 2009).

Dans cette prise en compte relativement tardive de la notion de patrimoine industriel dans la reconversion, le Royaume-Uni occupe une place à part. Premier pays où s'est développée la révolution industrielle, dès le XVIII ${ }^{e}$ siècle, il est aussi le premier pays qui doit faire face, dès la seconde révolution industrielle, à la fin du XIX ${ }^{\mathrm{e}}$ siècle, au déclin de l'industrie ancienne. Les premières actions de reconversion sérieuses ne remontent cependant pas avant l'Entre-deux-guerres, même si c'est très tôt par rapport aux autres pays industrialisés de l'époque. Dans ce mouvement, les préoccupations patrimoniales concernant l'industrie, si elles apparaissent tardives comparativement à celles concernant d'autres types de patrimoine (protégés dès la fin du XIX ${ }^{e}$ siècle pour certains bâtiments ou monuments remarquables), sont néanmoins particulièrement précoces par rapport aux autres Pays de Tradition Industrielle ${ }^{1}$ (Cartier, 2003). K. Falconer (2006) précise cependant que faire de ce pays l'unique pionnier du mouvement de protection du patrimoine industriel serait simplificateur.

$4 \mathrm{Si}$, après une cinquantaine d'années d'existence, les études et travaux retraçant l'historique, la vie et le développement du mouvement (Palmer, Neaverson, 1998: Falconer, 2006), voire les interrogations sur son futur (Cossons - éd, 2000 ; Casella, Symonds - éds, 2005) se multiplient, les travaux en français sont souvent plus anciens, plus rares, plus généraux ou plus illustratifs (Daumas, 1980 ; Andrieux, 1992 ; Belhoste, Smith, 1997; De Roux, 2000) et, surtout, dans les deux langues, l'approche géographique par les paysages et les territoires est rarement centrale.

Or, les paysages et territoires britanniques sont les témoins de la précocité de la prise en compte du patrimoine industriel, par l'intensité des reconversions patrimoniales, leur variété et leur nombre, même si la masse initiale des espaces à traiter laisse encore beaucoup de sites abandonnés (Deshaies, 2007). La précocité est aussi sensible dans l'élargissement spatial et thématique très fort, au Royaume-Uni, de la notion de patrimoine industriel, qui s'applique aujourd'hui à de vastes territoires. Elle lui donne ainsi une place à part dans ce domaine, même si l'on retrouve, notamment dans la dualité du traitement des sites, des constantes applicables à d'autres PTI.

6 Après avoir retracé le développement de la notion de patrimoine industriel dans un contexte international, ce travail s'attachera à montrer ensuite les différentes facettes et la spécificité de la prise en compte de ce patrimoine dans la reconversion au Royaume-Uni. L'élargissement spatial et thématique de la notion seront en particulier développés, autour de quelques exemples représentatifs et/ou emblématiques.

\section{I - L'évolution de la notion de patrimoine industriel dans le monde et au Royaume-Uni}

7 La notion de patrimoine industriel dérive de celle d'archéologie industrielle, développée au Royaume-Uni dès les années 1950. Ces deux notions sont donc récentes et ne se sont pas imposées de la même façon dans toute l'Europe et dans le monde. 


\section{A - Un concept jeune}

8 Le patrimoine industriel est l'un des derniers types de patrimoine reconnus, bien loin derrière les patrimoines religieux, civils, militaires, naturels, etc. Ce retard dans l'étude, la protection et la mise en valeur de l'industrie passée n'est pas seulement dû à la relative jeunesse de l'objet en question, certains sites remontant au XVIII ${ }^{e}$ siècle, voire au delà lorsqu'il s'agit de proto-industrie ${ }^{2}$. Il est en fait essentiellement lié à la perception négative, d'ailleurs assez récente, de l'industrie dans l'opinion. En effet, lors de l'apogée industrielle des XIX et début du XX $\mathrm{XX}^{\mathrm{e}}$ siècles, l'industrie bénéficiait d'une image plutôt positive puisqu'elle représentait le progrès et la modernité. Cartes postales et en-têtes magnifiées du courrier des entreprises célébraient alors l'usine comme le phare d'un monde nouveau.

9 La perception négative commence au milieu du $\mathrm{XX}^{\mathrm{e}}$ siècle et découle d'abord de la pollution, puis, avec la crise, de l'ambiance des sites abandonnés (on peut trouver un certain romantisme aux ruines d'un château médiéval, plus rarement à un hautfourneau rouillé). Dans le premier cas, la fermeture et la disparition de l'usine, en provoquant une amélioration de l'environnement et du cadre de vie, sont vues par certains riverains, évidemment non salariés de l'usine en question, comme des faits positifs.

$10 \mathrm{Au}$ delà de ces perceptions, la volonté de ne pas conserver le patrimoine industriel provient aussi de la pratique politique de l'usine. Au moment du déclin, puis de la fermeture, les luttes et les conflits opposant patrons conservateurs et syndicats de gauche ont souvent poussé les uns comme les autres à préférer détruire un site après la fermeture: la direction, comme la municipalité et beaucoup d'habitants, dans une phase de deuil bien compréhensible, décident, ou tout au moins poussent à la destruction de l'usine, symbole de passé révolu et d'échec économique. Ce rejet accompagne la volonté marquée des autorités locales de débarrasser le territoire d'une image grise et industrieuse censée repousser les investisseurs extérieurs. La destruction de l'ancien tissu industriel, voire la négation de l'histoire industrielle, semble alors le passage obligé vers une renaissance du territoire.

11 Cette phase de deuil est plus ou moins longue en fonction de la rapidité de la reprise économique, mais elle se caractérise toujours par une sorte de négation du passé industriel. Elle est très sensible dans tous les territoires industriels et en particulier dans les villes-usines ${ }^{3}$, nées de l'industrie. Il faut attendre le dépassement de cette phase de deuil pour accepter de redécouvrir le passé industriel et envisager une préservation d'un patrimoine industriel peu à peu perçu de façon plus positive, mais entre temps rouillé, abîmé, voire vandalisé. Enfin, cette perception positive croissante est aussi liée à la disparition rapide de l'industrie fordiste et de nombre de ses bâtiments. Cette raréfaction les rend plus intéressants aux yeux de la population.

Plus généralement, la préservation du passé, d'un patrimoine héritage des générations précédentes est aussi, dans le contexte de la mondialisation, un réflexe identitaire de protection face à une uniformité grandissante. Or, dans un quartier industriel, dans une ville-usine, dans une vallée ou un bassin industriel, l'usine, même fermée, fait partie de cette identité, surtout lorsque le territoire s'est construit autour d'elle. Sa protection et sa mise en valeur deviennent donc incontournable.

13 En outre, les sociétés occidentales, de plus en plus tertiaires, savent aujourd'hui faire fructifier ces héritages. Si la vieille usine n'attire jamais autant qu'un château médiéval 
ou une ruine antique, elle entre néanmoins dans une offre culturelle et complète les traces des autres époques.

Enfin, la conservation du patrimoine industriel entre parfaitement dans le cadre du développement durable, car la récupération d'anciens bâtiments ou d'anciens terrains industriels permet de ne pas étendre la ville aux dépens de l'agriculture, tout en réduisant les coûts liés à une périurbanisation toujours plus éloignée des centres.

\section{B - Au Royaume-Uni, une protection précoce du patrimoine industriel}

15 L'image de l'industrie dépend aussi de la place que le travail a tenu et tient dans la société. Il semble que, dans les pays catholiques, le plus souvent latins, la valeur travail soit moins valorisée qu'en pays protestants où la réussite professionnelle est signe de la bienveillance divine (Leboutte, 1997). Ceci peut expliquer en partie la valorisation de l'histoire industrielle dans les pays de la Réforme, avec par exemple la protection précoce observée au Royaume-Uni, dans certaines parties de l'Allemagne, ou en Suède. Mieux encore, au Royaume-Uni, l'industrie est associée à l'apogée économique du pays. Sa préservation permet donc "de célébrer la prospérité de l'ère victorienne où, grâce à son avance dans l'industrialisation, la Grande-Bretagne était devenue la puissance mondiale dominante» (Andrieux, 1992). La préservation du patrimoine industriel débute donc dans ce pays où est née la grande industrie. C'est aussi dans ce pays que les usines sont les premières à fermer, parfois dès la seconde révolution industrielle, fatale à certains sites enclavés de la première. Si la date officielle de la définition de la notion de " monument industriel " par le Council for British Archeology, est 1959, des exemples de conservation existent dès l'entre deux guerres, notamment par des démarches privées ou associatives (Andrieux, 1992 ; Falconer, 2006).

Comme c'est souvent le cas au Royaume-Uni, l'enthousiasme local précède, puis accompagne, les initiatives régionales ou nationales, telle l'enquête The Industrial Monuments Survey, mise en place en 1963, recensement essentiel avant d'envisager une quelconque protection. Ainsi, les premiers travaux concernant le patrimoine industriel sont souvent des études de cas réalisées par de multiples sociétés et associations locales. Ces travaux, fréquemment liés à des vestiges de la première révolution industrielle, voire de la proto-industrie, s'effectuent sous forme de fouilles dégageant les ruines d'un bâtiment, un haut-fourneau du XVIII ${ }^{e}$ siècle par exemple. Le terme consacré à cette époque est donc celui d'archéologie industrielle, utilisé pour la première fois dans les années 1950 par M. Rix (Falconer, 2006; www.industrialarcheology.org.uk). Peu de temps après, K. Hudson, historien à l'université de Bath (Chassagne, 2002 ; Falconer, 2006), reprend l'expression, publie en 1963 un ouvrage de référence, Industrial archeology : an introduction, fonde une revue, le Journal of Industrial Archeology en 1964, puis, en 1973, l'Association for Industrial Archeology (AIA). Publiant, depuis 1976, l'Industrial Archeological Review, l'AIA fédère de nombreuses sociétés locales (60 en 2008) et permet de centraliser un très grand nombre d'informations et d'études anciennes ou récentes sur le sujet. Son siège est à Ironbridge, lieu symbolique du patrimoine industriel mondial (cf. partie II).

Les publications et études plus générales se multiplient ensuite, comme celles de Hudson (1971), Buchanan (1972), Cossons (1975) ou encore Falconer (1980) aux côtés d'une pléthore d'ouvrages et d'articles thématiques ou régionaux. Dans les années 1980, 
le mouvement d'inventaire et de protection s'accélère, en réponse à des démolitions sauvages de bâtiments (Falconer, 2006) et avec la création, dans le cadre de la loi sur le

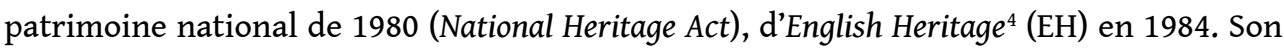
objectif est à la fois l'étude et l'archivage des sites, la gestion, parfois partagée avec National Trust ${ }^{5}$, de certains sites et le conseil au gouvernement en matière de patrimoine. Il est par exemple responsable de la gestion du site de l'Ironbridge déjà évoqué.

18 La création d'EH s'inscrit en fait dans le boom patrimonial des années 1980, observé suite à la loi de 1980. En effet, face à la crise de l'industrie fordiste et aux nécessités de la reconversion, les gouvernements Thatcher et Major ont mis le patrimoine au cœur de leur politique de redéveloppement régional. Dans les Régions de Tradition Industrielle (RTI), ce patrimoine est bien évidemment très souvent lié à l'industrie. La transformation, à coup de subventions gouvernementales massives, de mines en musées ou, au delà, de quartiers industriels entiers en zones commercialo-culurelles ${ }^{6}$, a souvent eu pour objectif premier d'assurer rapidement des emplois et des bénéfices commerciaux, sans respect scrupuleux pour le passé et l'histoire industriels (Nenadic, 2002). Ces actions, très critiquées dans les décennies suivantes pour leur manque de vision historique globale (notamment dans le cas des mines) ou encore pour leur côté clinquant, fourre-tout et historiquement peu véridique (grandes opérations de renouveau urbain), ont creusé un fossé entre professionnels du patrimoine et historiens universitaires. Les premiers sont ainsi restés plus attachés aux objets et aux bâtiments, pouvant être exposés ou visités facilement qu'à l'histoire globale d'un site patrimonial et des ses acteurs. Ce débat rejoint celui existant entre preservation et conservation (Cullingworth, Nadin, 2006) ; la première implique le maintien de l'état original, la seconde accepte modifications et améliorations de cet état orignal. Ainsi, en fonction de la précocité du mouvement et de ces vifs débats, l'expression « archéologie industrielle» reste-t-elle plus utilisée au Royaume-Uni que celle de "patrimoine industriel », largement plus répandue dans le reste du monde.

\section{C - Diffusion et évolution de la notion}

19 L'expression "archéologie industrielle » est reprise aux États-Unis avec en 1972 la fondation de la Society for Industrial Archeology, puis sur le continent européen en Flandre belge (création de la Vlaamse Vereniging vor Industriële Archeologie en 1978) ou encore en France, avec la parution de l'ouvrage fondateur de M. Daumas en 1980, «L'archéologie industrielle en France ». En 1979, est fondée la revue du même nom, publiée par le Comité d'Information et de Liaison pour l'Archéologie, l'étude et la mise en valeur du patrimoine industriel (CILAC) né la même année.

Durant les années 1980, les pays latins, comme l'Italie ou l'Espagne, connaissent aussi ce mouvement. Enfin, plus récemment, les pays d'Europe Centrale et Orientale découvrent la notion et leur intégration récente dans l'UE devrait y favoriser son développement. Il est d'ailleurs déjà très sensible dans des pays qui ont connu, sur des portions non négligeables de leur territoire, les révolutions industrielles comme la Pologne ou la République Tchèque. La Pologne possède ainsi un site minier inscrit sur la liste du patrimoine mondial et la ville de Łódź, « Manchester de l'Est » et haut lieu de l'industrie textile de l'ancien Empire russe, tient largement compte du patrimoine dans sa reconversion, tant les usines en constituaient le squelette. 
Par contre, plus à l'est encore, notamment en Russie, la tâche est immense. S'il existe bien une association russe affiliée au TICCIH (cf. III), la MPAS (Moscow Architecture Preservation Society - Association de protection de l'architecture moscovite), son objectif est à la fois thématiquement large, dépassant de loin la préservation du seul patrimoine industriel, et géographiquement réduit, puisqu'il ne concerne que la région de Moscou. Parallèlement, l'expression "archéologie industrielle » devient, dès les années 1980, insuffisante car trop réductrice face au développement de la nouvelle activité. En effet, celle-ci s'étend très vite, au delà des fouilles, à l'étude des objets et des techniques, puis des bâtiments industriels encore debout et de leurs annexes et, enfin, de plus vastes territoires encore, abordés dans cette perspective patrimoniale dans la troisième partie de cet article. L'expression « patrimoine industriel » finit donc par s'imposer vers la fin des années 1980, tout au moins sur le continent car le Royaume-Uni, pour les raisons évoquées plus haut, reste plutôt fidèle à l'expression "archéologie industrielle »; le concept y est cependant tout aussi englobant que sur le continent.

23 Après une prise de conscience globalement récente, mais où le Royaume-Uni joue un rôle de précurseur, la nécessité de protéger le patrimoine industriel est acceptée aujourd'hui, même si elle n'est pas encore comprise par tout le monde, notamment sous la pression foncière ou en temps de crise. Cette protection se traduit sur le terrain britannique par des actions particulièrement variées et de plus en plus larges.

\section{II - Le patrimoine industriel britannique : protection intégrale et/ou indirecte}

24 La patrimonialisation accompagne donc aujourd'hui la majorité des démarches de reconversion, mais de façon duale, ce qui n'est d'ailleurs pas une spécificité britannique, même si c'est très marqué dans ce pays en raison justement de la précocité de la notion dans le domaine industriel. Cette dualité est souvent liée aux vifs débats évoqués plus haut entre conservation et preservation.

La première facette de cette dualité est une prise en compte intégrale du patrimoine industriel dans la reconversion. Elle est alors effectuée dans un but pédagogique en conservant les caractéristiques propres de l'objet, de l'usine, du site en en faisant un témoin édifiant d'une époque révolue. La fonction du site, après avoir été industrielle, devient donc culturelle et, au delà, touristique.

L'autre facette est une prise en compte indirecte du patrimoine industriel dans la reconversion, en l'intégrant dans des opérations à finalité commerciale, administrative, culturelle (autre que le but pédagogique évoqué ci-dessus), sportive, de loisirs, résidentielle, etc. Dans ce cas, le patrimoine industriel est préservé (en partie ou totalement), mais pas en tant que tel, seulement comme une des composantes, parfois essentielle, de la reconversion. 


\section{A - La préservation intégrale : des musées et des parcs industriels très (trop ?) nombreux}

\section{1 - De nombreux petits musées} transformés en musées, dans un objectif de rentabilité rapide et de réemploi, les mines de charbon fermées ré-ouvrant "peu après sous forme de mémoriaux de la mine et du charbon, les anciens mineurs étant employés comme guides » (Nenadic, 2005).

Ce type de reconversion revient donc à une prise en compte totale du patrimoine industriel. Elle touche souvent en premier lieu des éléments proto-industriels relativement discrets (photo 1), dans le cadre de la nostalgie, voire de la fascination pour la vie passée et traditionnelle de l'Angleterre préindustrielle, dans un mouvement de retour aux sources, "grass roots ", évoqué par Falconer (2006).

Photo 1 : Les maisons-ateliers des tisserands d'Arlington Row à Bibury, South-West ${ }^{7}$ (Edelblutte, 2002)

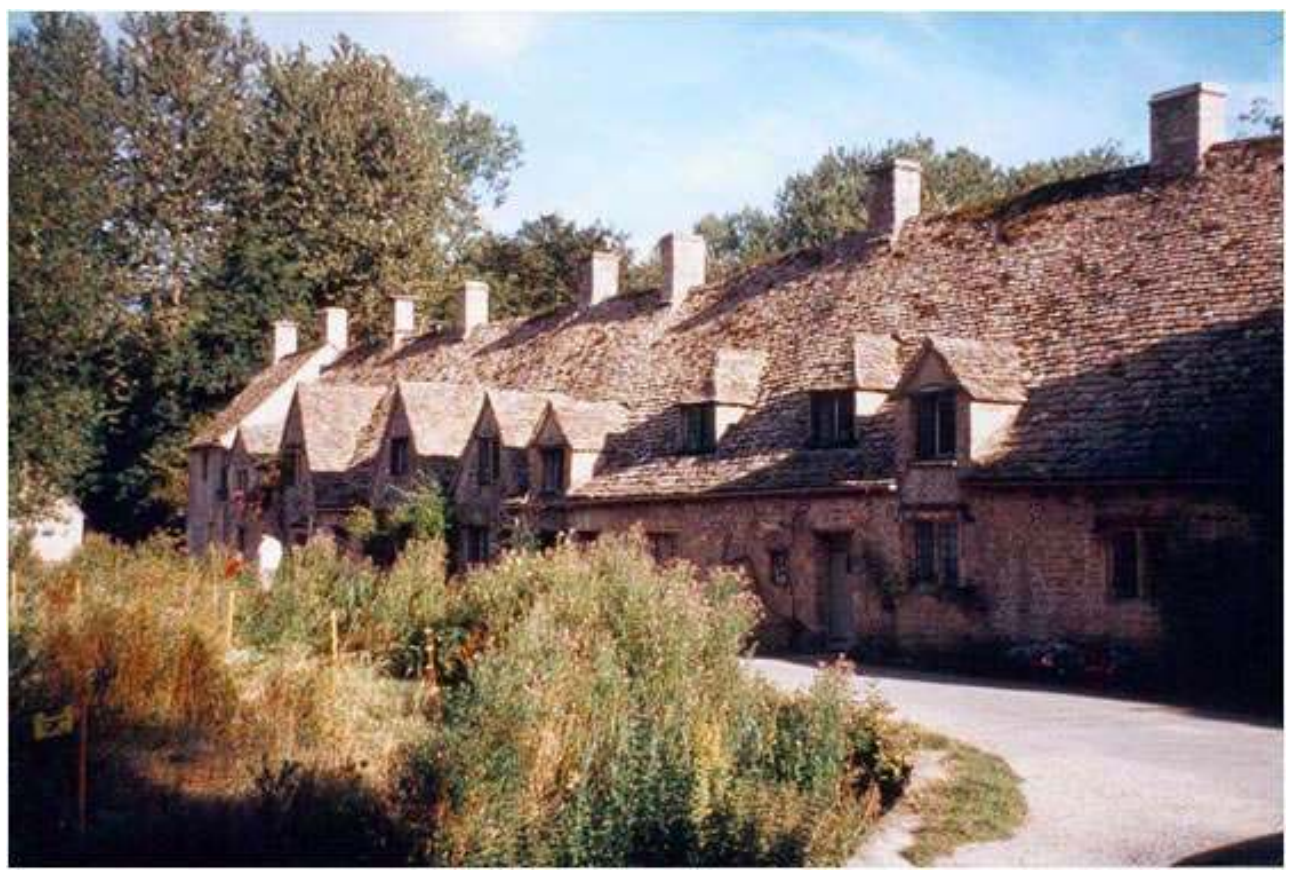

Construites au XVII e siècle, elles abritaient autrefois des métiers à tisser dans leurs hauts combles et le logement en rez-de-chausée. Liées à l'Arlington Mill, moulin voisin où l'on traitait les tissus, elles forment un petit village proto-industriel qui est l'un des plus visités de la région touristique des Costwolds.

La muséification touche aussi de nombreux éléments plus purement industriels. Cela concerne le plus souvent une mine ou une usine de taille modeste, mais parfois des sites plus importants, comme des usines tubulaires ${ }^{8}$ (sidérurgie, chimie...). Au delà de cette muséification totale, une patrimonialisation populaire est celle de très nombreuses brasseries, ou encore distilleries de whisky en Écosse, complétées par un musée alors qu'elles sont toujours en fonction.

30 Ces petits musées posent cependant un grave problème de rentabilité, notamment lorsque l'activité de visite et de découverte est une activité unique sur le site. Aucun de ces musées ne figure évidemment dans les lieux les plus visités des régions 
britanniques (www.tourismtrade.org.uk). De plus, la concurrence entre diverses autorités locales (comtés, autorités unitaires, municipalités) peut amener à des réalisations similaires très proches et donc fatalement peu rentables?.

\section{2 - L'ère des parcs à thème industriel}

Face à ces difficultés, la tendance est à la réalisation de véritables parcs à thèmes industriels, mettant en réseau, localement, plusieurs musées et attractions liées à l'industrie et à ses à-côtés.

Figure 1 : L'Ironbridge Gorge Park

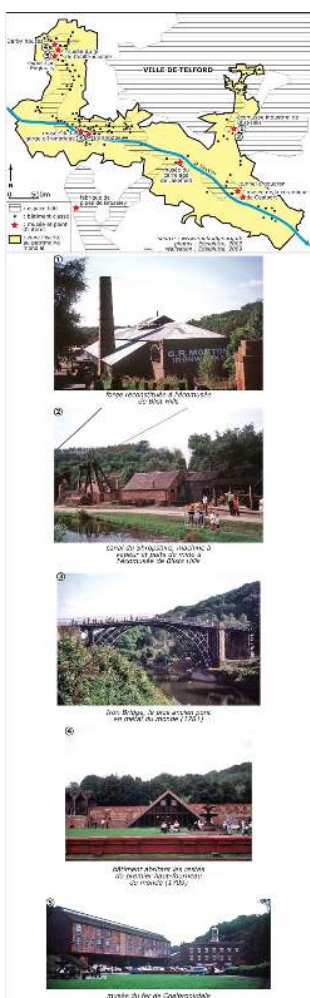

L'exemple emblématique en est l'Ironbridge Gorge Park (West Midlands) présenté sur la figure 1. Le site est organisé autour du premier pont métallique du monde (l'Iron Bridge) et du premier haut-fourneau produisant de la fonte au coke réalisé par A. Darby en 1709. L'entreprise qui a suivi, la $C^{\text {ie }}$ Coalbrookdale, fonctionne environ pendant 250 ans ( $\mathrm{du}$ début $\mathrm{du} \mathrm{XVIII}^{\mathrm{e}}$ siècle au milieu $\mathrm{du} \mathrm{XX}^{\mathrm{e}}$ ), tout en conservant une collection d'objets produits durant cette période ; objets qui sont valorisés aujourd'hui dans des musées. Tout un ensemble industriel (sous-traitants, clients, fournisseurs, etc.) naît autour de cette compagnie, se développe et ferme progressivement entre 1912 et 1950.

Dès 1967, une association est créée autour de la mise en valeur du patrimoine industriel et réussit à préserver, puis à valoriser cet ensemble d'environ $15 \mathrm{~km}^{2}$, dont les composantes principales sont :

- le musée du fer, installé dans une ancienne usine en face du fameux haut-fourneau de

Coalbrookdale ;

- l'Iron Bridge, premier pont en fonte au monde, construit entre 1779 et 1781 ;

- les fours d'une fabrique de porcelaine et un musée du carrelage à Coalport ; 
- l'écomusée de Blists Hills, parc de plein air mélangeant des parties préservées (hautsfourneaux, installations du canal de Shropshire, avec un plan incliné) et des parties recréées à partir de bâtiments, d'outils et de matériel lourd récupérés ailleurs et transférés ici. Le tout est couplé à un village ouvrier du XVIII ${ }^{e}$, avec ses échoppes et ses habitants, incarnés par le personnel du parc. Cette mise en réseau de musées proches et complémentaires, liés aux vestiges et au parc de Blist Hills, permet une offre touristico-culturelle plus diversifiée et donc plus attractive. Ainsi, l'Ironbridge Gorge Park accueille plus de 600000 visiteurs annuels (www.erih.net) en 2006 (pour 250000 visiteurs en 2001) ce qui en fait un des sites les plus visités des West Midlands.

Le Beamish Open Air Museum (North-East) est un autre exemple de parc industriel, même s'il ne s'agit pas ici de la mise en réseau de musées divers, mais d'une véritable et entière reconstitution, là où Blists Hills mêle vestiges locaux et reconstitutions. Centré sur la mine et la vie traditionnelle au XIX ${ }^{\mathrm{e}}$ siècle, il s'étend sur 120 ha et mélange, sur un site vierge (ce qui le différencie de l'Ironbridge) des vestiges de l'extraction charbonnière transférés de friches locales et des reconstitutions. Le parc, ouvert en 1972, est devenu le deuxième site touristique payant le plus visité du North-East en 2006 avec un peu plus de 320000 visiteurs annuels (www.tourismtrade.org.uk) et il s'auto-finance pratiquement entièrement grâce aux revenus des entrées.

Cette réussite montre que le patrimoine industriel est ici réellement une question d'identité locale plus que d'authenticité. En effet, le fait que le parc soit une reconstitution de diverses époques industrielles et minière et qu'il ne soit pas sur un ancien site industriel ou minier, n'a pas rebuté le public, même si cela a choqué les puristes et certains historiens qui considèrent que la perception du passée par le public est compromise (Nenadic, 2005).

Le Royaume-Uni est ainsi le pays d'Europe qui possède le plus de musées industriels ou à connotation industrielle. Le site de l'European Route of Industrial Heritage (www.erih.net) en a recensé 210, contre 197 en Allemagne (à l'origine de, et où siège l'ERIH) ou encore 50 en France. Certes, le décompte est partiel et lié à la volonté d'inscription des sites, mais le nombre important de sites britanniques inscrits est évidemment lié à la fois à la profondeur historique de l'industrialisation du pays et aux politiques de reconversion des années 1980/90.

La préservation intégrale, c'est-à-dire dans un but pédagogique, du patrimoine industriel a donc été utilisée durant les années 1980/90 comme un élément phare de la reconversion, d'abord sous la pression économique puis, plus récemment, dans un mouvement identitaire sensible et visible surtout dans les RTI britanniques (Sud du Pays-de-Galles, North-East, North-West, Yorkshire, West Midlands, Lowlands d'Écosse) se définissant en contrepoint de la réussite tertiaire du grand Sud-Est autour de Londres. Cette avance dans la valorisation du patrimoine a aussi permis au RoyaumeUni d'être le premier (avant même l'Allemagne pourtant très en avance dans la Ruhr) à expérimenter la mise en réseau de sites, sur des périmètres plus vastes, constituant de véritables parcs à thèmes industriels, comme celui de l'Ironbridge. Cependant, cette valorisation patrimoniale intégrale n'est pas possible, ni souhaitable partout, notamment en raison de la quantité des sites industriels abandonnés ; d'autres formes de mise en valeur patrimoniale existent donc. 


\section{B - Le patrimoine dans d'autres actions : une préservation indirecte}

39 Avec l'apparition de la notion de développement durable, qui pousse au renouveau urbain plutôt qu'à l'extension urbaine et à la rénovation (destruction/reconstruction), la réhabilitation des bâtiments industriels abandonnés à des fins autres que la muséification est visible dans tous les PAI. Cette préservation patrimoniale indirecte est particulièrement présente au Royaume-Uni en raison à la fois de l'importance du stock d'éléments industriels abandonnés et de la place de l'industrie dans l'histoire et l'identité de certaines régions.

\section{1 - Des actions souvent modestes}

Cette récupération est d'abord souvent limitée à de petits éléments de décor ou à des ruines, notamment en milieu rural, plus ou moins bien valorisées. Wagonnets miniers, locomotives industrielles, roues, outils et machines diverses sont installées à un carrefour, au milieu d'une place. Ce genre d'éléments est fréquent dans les villes industrielles, cités préexistantes aux révolutions industrielles et dont l'industrie n'a été présente que dans une partie du tissu urbain; partie néanmoins parfois très importante.

41 Ainsi, à Salford, ancienne banlieue industrialo-portuaire de Manchester (North-West), en prise directe avec le Manchester Ship Canal à grand gabarit reliant la ville à la mer, il ne reste qu'un pont tournant et quelques vestiges reconstitués, quelques panneaux pour rappeler l'ancienne occupation du quartier aujourd'hui totalement rénové et accueillant bureaux, résidences, musées et centres culturels et commerciaux à l'architecture très contemporaine (planche 1 ). 
Planche 1 : Quelques vestiges de la période industrialo-portuaire à Salford, dans le Grand Manchester (North-West)

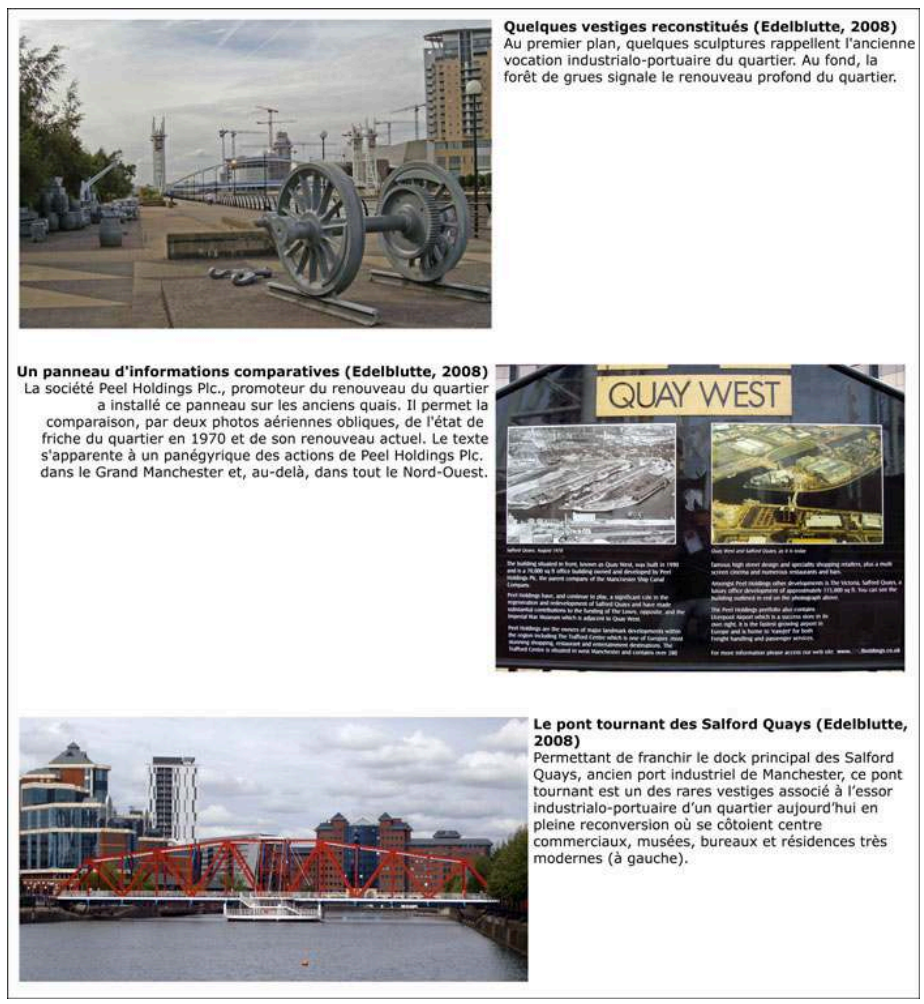

42 La valorisation du patrimoine industriel est en général beaucoup plus poussée dans les villes-usines où l'industrie était plus consubstantielle de l'identité et du paysage local. Sa patrimonialisation est donc plus essentielle et prend des formes à la fois plus variées et plus imposantes, souvent liées au commerce et à la résidence.

\section{2 - Commerce et résidences, une valorisation commode et donc fréquente}

43 Une des reconversions patrimoniales les plus courantes est, en lien avec la tertiarisation particulièrement poussée de la société britannique, dirigée vers le commerce. Cette reconversion est facilitée par la faiblesse de l'offre commerciale des villes-usines nées autour d'un cœur minier et/ou industriel et où tout était sacrifié à la production. Elle est facilitée lorsque que le bâtiment industriel est une usine à étage ou à sheds ${ }^{10}$ et non une usine tubulaire, plus difficilement réutilisable.

Les exemples sont nombreux dans les anciennes usines textiles, de la Salt's Mill à Saltaire (Yorkshire \& The Humber) à la Masson Mills à Matlock Bath (East Midlands) en passant par une des plus belles réussites en termes de fréquentation, la Moscow Mill d'Oswaldwhistle, à Accrington dans le Lancashire (North-West). Cette usine construite en 1824, relayant dans un mouvement classique, les ateliers implantés autrefois à domicile, a été convertie par des intérêts privés ${ }^{11}$ en un vaste centre commercial, accueillant plus de 80 boutiques et des artisans, représentant 225 emplois dans 5 anciens bâtiments industriels. Une petite partie du site est réservée à un musée textile retraçant les grandes étapes de l'histoire du site et la vie quotidienne des ouvriers. L' Oswaldswhistle Mills Shopping Village est, grâce à cette reconversion, le second site industriel patrimonial visité du Royaume-Uni avec plus de 550000 visiteurs annuels en 2007 (www.tourismtrade.org.uk), ce qui le place juste derrière l'Ironbridge Gorge Park. 
Il existe néanmoins une différence de taille puisque si ce dernier est bien une valorisation patrimoniale directe et intégrale, l'Oswaldswhistle Mills Shopping Village est une valorisation patrimoniale indirecte et sa fréquentation est due non pas au caractère industriel du site, mais surtout au fait qu'il abrite un centre commercial. Il s'agit donc d'une mise en valeur plus incomplète et moins scientifiquement satisfaisante qu'un musée ou même qu'un parc à thème industriel, mais cela a permis la conservation, dans le tissu urbain, dans le paysage et donc dans le quotidien des habitants, de l'empreinte industrielle majeure que constituait cette ancienne usine textile.

Si la réoccupation par des commerces est fréquente, les anciens bâtiments industriels peuvent être préservés à d'autres fins, dont l'une des plus fréquentes est la transformation en résidences. En effet, dans de nombreuses villes-usines mais aussi dans des villes marquées par une forte industrialisation, ce qui est le cas de la plupart des grandes agglomérations du Nord anglais et des West Midlands, la présence très importante, dans le tissu urbain préexistant, de bâtiments industriels abandonnés a poussé vers une reconversion résidentielle. Celle-ci est favorisée, dans le cas d'usines textiles ou d'entrepôts en briques, à étages et à larges et nombreuses ouvertures (la lumière étant essentielle dans le travail du textile) et non tubulaires à l'architecture plus spécifique. La hauteur importante de plafond, le cachet architectural et les larges ouvertures, amènent souvent à la création de lofts, vastes appartements à pièce unique destinées à des populations plus aisées. Ce type de transformation est très présent dans les villes du Lancashire, à Manchester, dans l'ouest du Yorkshire (Leeds, Bradford) ou encore à Newcastle-upon-Tyne dans le North-East (photo 2). 
Photo 2 : Une reconversion résidentielle dans une ancienne usine à Newcastle-upon-Tyne, NorthEast (Edelblutte, 2008)

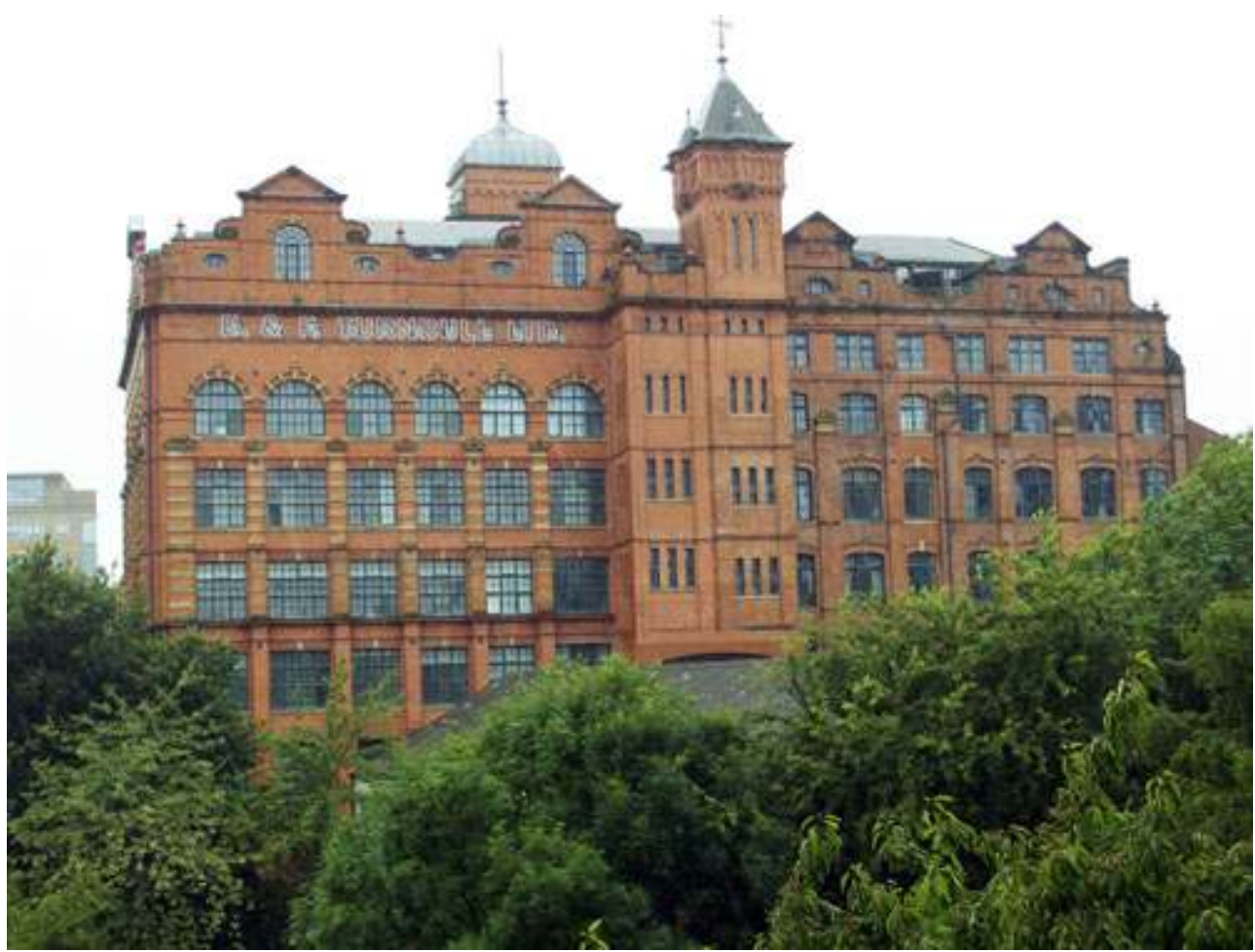

Construit en 1888 pour l'imprimerie Robinson \& Co., agrandi en 1898, transformé ensuite en entrepôt par E. \& F. Turnbull, ce bâtiment à étages et en brique, typique des usines anglaises de l'époque est situé au contact du centre-ville ancien de Newcastle. De plus, il domine la vallée de la Tyne, offrant donc de larges points de vue. En 2002, il est reconverti en lofts, vastes appartements de luxe conservant et valorisant de nombreux éléments industriels d'origine (poutres et piliers métalliques notamment).

La reconversion résidentielle, rarement envisagée pour des raisons de coûts (dépollution, mise aux normes, etc.) dans les premiers temps du déclin du fordisme, est donc possible dans le cas de bâtiments industriels proches des centres-villes, où les prix de l'immobilier sont élevés. Elle est particulièrement bien représentée au Royaume-Uni où le potentiel de départ est très large.

\section{3 - D'autres formes de reconversion patrimoniale}

Au delà des reconversions commerciales et résidentielles, d'autres formes de valorisation patrimoniale plus originales existent.

Les grosses usines ou entrepôts liés aux révolutions industrielles, s'ils se prêtent bien à la reconversion en espaces commerciaux, peuvent aussi être utilisés comme supports d'éléments culturels. L'offre culturelle, notamment dans les grandes villes-usines et villes industrielles, entièrement consacrées à l'industrie ou presque, était en effet faible. La récupération des anciens et vastes bâtiments industriels, mais surtout des docks et entrepôts, peut ainsi être une opportunité bienvenue qui allie une nouvelle offre culturelle en conservant une part de l'ancienne identité de la ville ou du quartier. Si l'exemple le plus célèbre, celui de la Tate Modern installée dans une ancienne centrale électrique thermique, se situe à Londres, sur les bords de la Tamise, d'autres existent un peu partout au Royaume-Uni, notamment dans les RTI (planche 2). 
Planche 2 : Exemples de reconversion culturelle valorisant le bâtiment industriel ou portuaire

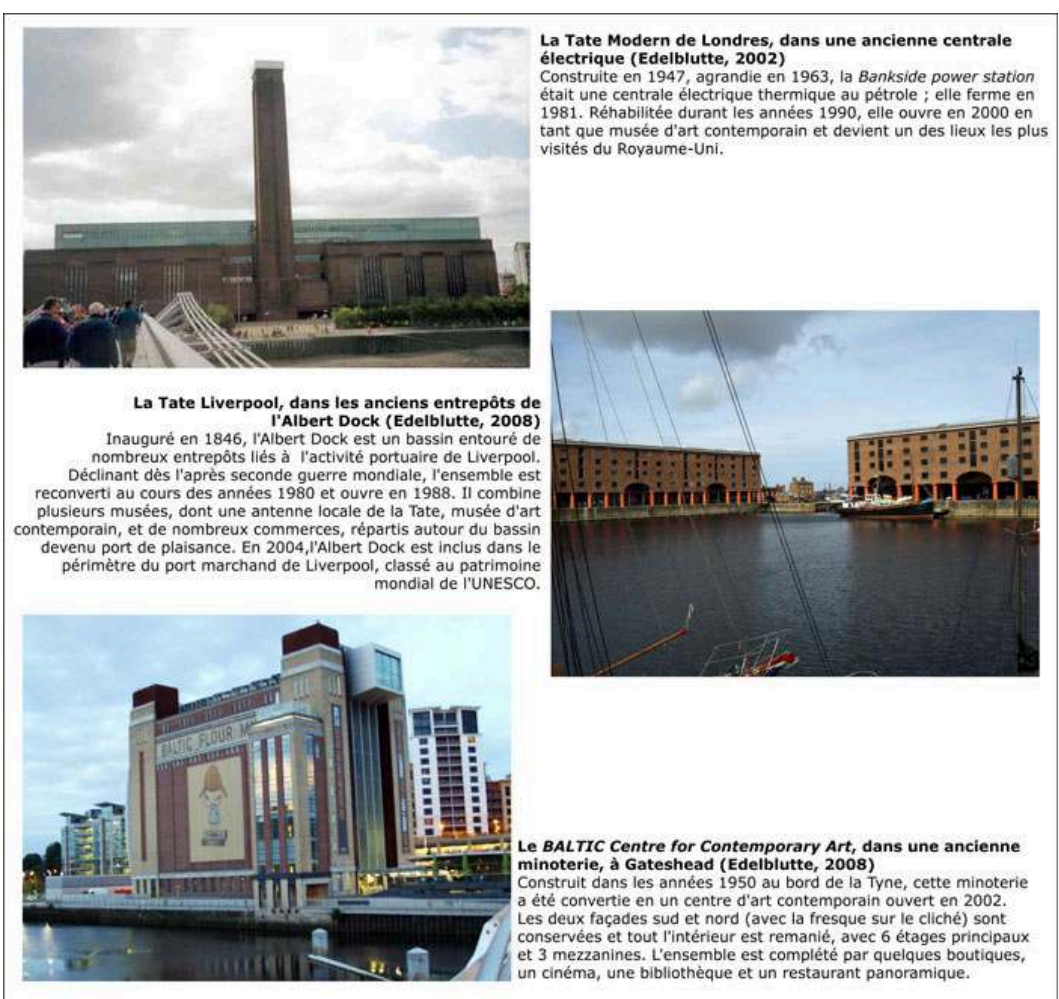

49 En réalité, la variété des types de reconversion intégrant une composante patrimoniale est quasi infinie. Les grandes usines en brique peuvent ainsi accueillir des bureaux, des activités de loisirs, des centres sociaux, des pubs (photo 3), etc. voire d'autres industries, notamment des PMI. 
Photo 3 : Le pub RA!N à Manchester (North-West), une ancienne usine de parapluies (Edelblutte, 2008)

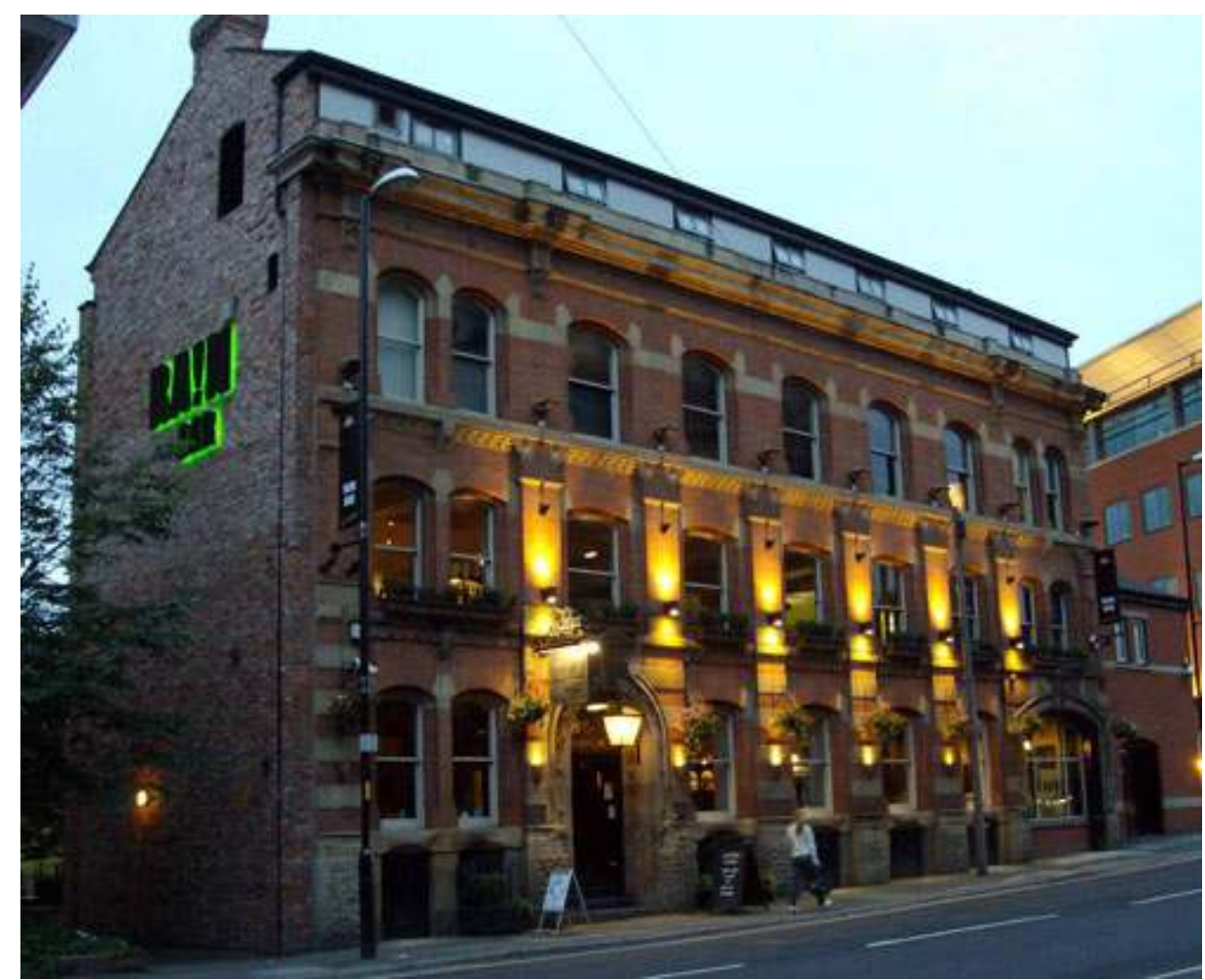

Ce bâtiment de style victorien abritait une usine de parapluies. Longtemps abandonné, il ré-ouvre en 1999 sous forme d'un pub restaurant de 3 étages, avec, à l'arrière, une terrasse dominant le Rochdale Canal ouvert en 1804 à travers la Chaîne Pennine et aujourd'hui réhabilité.

50 La préservation patrimoniale d'un bâtiment ne passe donc pas forcément par la réalisation d'un musée. Mieux encore, l'intégration d'une nouvelle activité dans les habits de l'ancienne, si elle choque parfois les puristes et avive les tensions entre historiens et professionnels du patrimoine, est souvent la meilleure garantie de la conservation d'un patrimoine vivant. Cette tendance à la constitution d'un patrimoine industriel vivant et actif, est également sensible avec l'élargissement spatial de la notion et sa mise en réseau, au delà de frontières du Royaume-Uni.

\section{III - Élargissement et mise en réseau du patrimoine industriel britannique}

51 La notion de patrimoine industriel, s'inscrivant dans l'élargissement de la notion globale de patrimoine évoqué dans l'introduction, touche aujourd'hui des thématiques de plus en plus larges et des territoires de plus en plus vastes, dont la gestion est évidemment plus complexe. Les territoires industriels, hérités ou actifs, s'entendent en effet à plusieurs échelles (figure 2). Cet élargissement, visible notamment au niveau des sites industriels classés au patrimoine mondial, s'accompagne d'une mise en réseau au niveau européen et au niveau mondial. 
Figure 2 : L'emboîtement des territoires industriels à différentes échelles

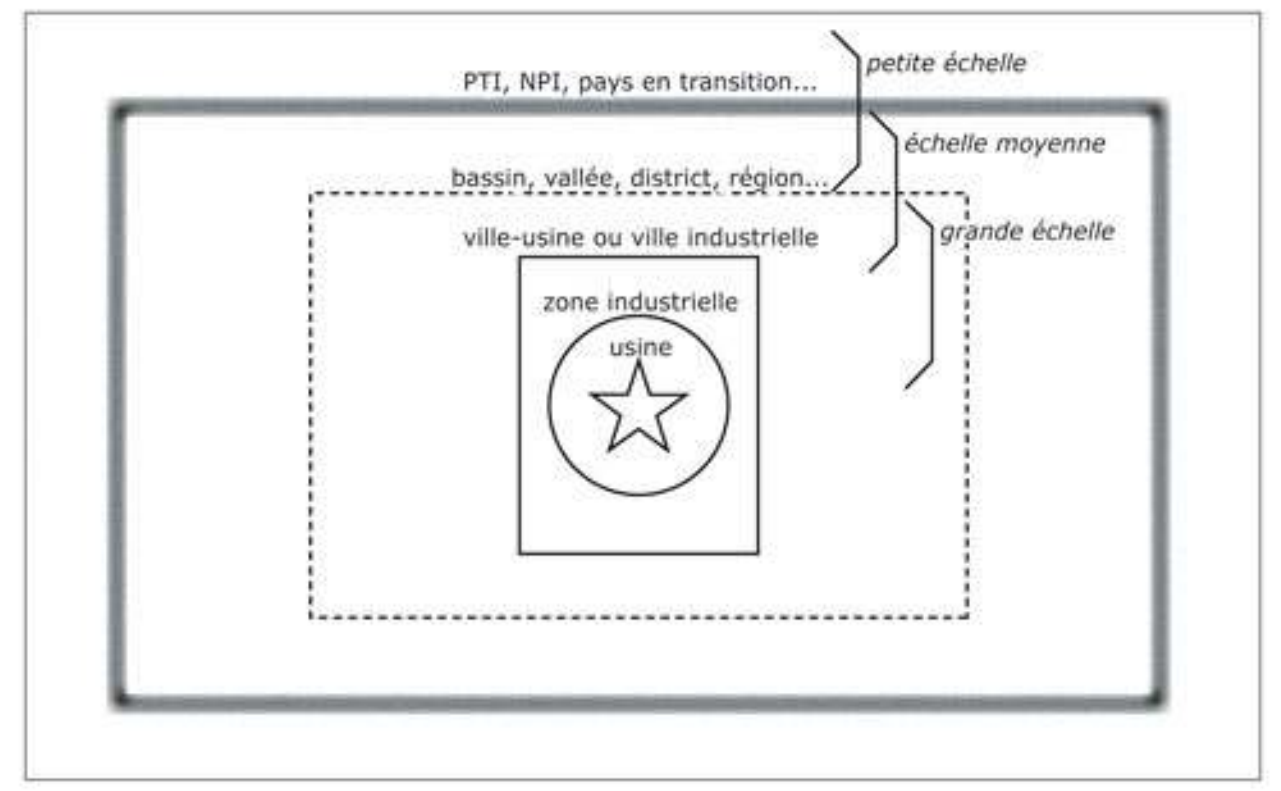

\section{A - Les villes-usines, un territoire patrimonial emblématique}

52 Au delà du site lui-même, le premier territoire industriel à avoir été mis en patrimoine est la ville-usine. Les ensembles les plus anciens ou les plus aboutis ont été préservés en premier, alors que d'autres villes-usines, plus récentes ou moins bien préservées, sont seulement en cours de protection.

New Lanark, en Ecosse, est ainsi préservée en tant que représentative des premières villes-usines du début du XIX siècle. Cet ensemble a été fondé en 1785 au Sud de Glasgow, et remodelé par l'industriel philanthrope Robert Owen entre 1809 et 1824 . Ce sont ses réalisations qui sont considérées comme exemplaires, Robert Owen a voulu développer ici une société idéale, sans crime, sans pauvreté et sans misère, en augmentant la taille et le confort des logements ouvriers et en les éduquant, espérant ainsi les responsabiliser, les motiver et augmenter leur productivité. New Lanark regroupe donc tous les éléments clés de la ville-usine, de l'usine aux bâtiments économiques et sociaux en passant par les cités ouvrières. L'usine ferme en 1968 et le site est mis sous la gestion du New Lanark Conservation Trust, fondation d'utilité publique à but non lucratif. Celui-ci décide alors de restaurer le site à son âge d'or, c'est-à-dire la première moitié du XIX ${ }^{\mathrm{e}}$ siècle, en détruisant donc les bâtiments postérieurs. Ce choix est évidemment critiqué par certains et peut être mis en lien avec le débat entre professionnels du patrimoine et historiens.

Un autre exemple emblématique, pour sa grande homogénéité et sa représentativité de l'aboutissement ultime du paternalisme, est celui de l'ancienne ville-usine textile de Saltaire (figure 3), près de Bradford (Yorkshire \& The Humber).

Cet extraordinaire ensemble industrialo-urbain est né en 1853 de la volonté de Titus Salt, qui voulait transférer ses 5 usines textiles du centre de Bradford dans une seule installée en bordure d'une voie ferrée et d'une rivière navigable. Il s'agissait aussi, pour ce patron éclairé, de fournir aux ouvriers très mal logés dans des immeubles insalubres 
à Bradford, des logements décents et salubres. Ces logements sont complétés par les inévitables bâtiments économiques et sociaux (réfectoire, bains, hôpital, école, institut - regroupant plusieurs activités sociales, maisons réservées aux retraités, église, etc.), le tout formant une ville-usine complète pour les 3 à 4000 employés de l'usine.

L'ensemble se délite progressivement, notamment à partir de 1933 lorsque les logements sont vendus peu à peu à leurs occupants, et ensuite, tout au long des années d'après guerre, avec le transfert progressif des activités de l'usine vers d'autres sites, voire vers l'étranger.

L'usine finit par fermer en 1986 ; rachetée par un particulier passionné, elle abrite, dès 1987, des expositions, ce qui permet sa préservation qui n'aurait pas été assurée en cas d'abandon.

En 1971, dès avant la fermeture, le bâtiment et, au delà, la ville-usine entière, sont classés et protégés par une conservation area, zone à l'architecture particulière et/ou à l'intérêt historique méritant une protection et un entretien. En 1985, 99,5\% des bâtiments sont inscrits sur la liste des bâtiments d'intérêt architectural ou historique particulier, à divers niveaux : grade I (église), II* (Salts Mill - usine principale - institut et école) ou II pour pas moins de 800 autres bâtiments de la ville-usine, essentiellement les cités ${ }^{12}$.

Cette évolution douce a permis le maintien de l'homogénéité de l'ensemble qui fait, avec son ancienneté et sa taille, son originalité par rapport à d'autres exemples contemporains du même type au Royaume-Uni (Walkerburn en Écosse) ou ailleurs en Europe (Cité Dollfuss de Mulhouse en Alsace).

Figure 3 : Une ancienne ville-usine patrimonialisée dans son ensemble : Saltaire (Yorkshire \& The Humber)

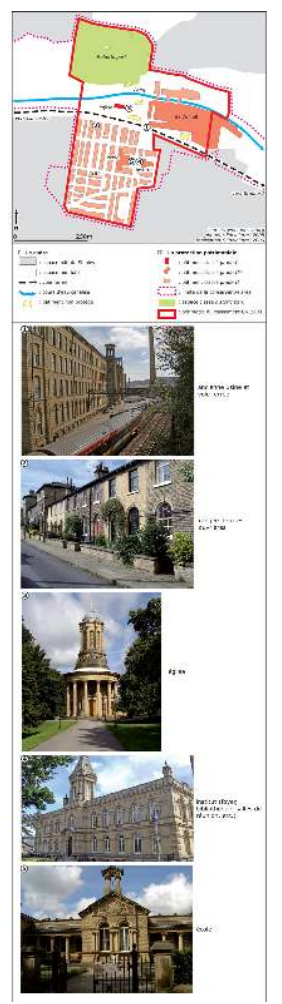


60 Enfin, une des particularités essentielle de Saltaire réside dans le fait que tout cet ensemble est vivant. En effet, aucun bâtiment n'est vide ; l'usine principale (Salts Mill, sur la rive droite de l'Aire) abrite des magasins, des antiquaires et des galeries d'exposition dans sa partie ouest ainsi que des commerces et nouvelles industries légères dans sa partie est. L'usine annexe (New Mill, sur la rive gauche de l'Aire) abrite les bureaux des services de santé de la ville de Bradford et des lofts.

61 Les anciennes cités sont toutes réoccupées et les anciens bâtiments économiques et sociaux également; l'institut, ancien bâtiment qui regroupait diverses activités sociales, conserve cette vocation; l'hôpital est devenu une crèche, les maisons des retraités sont devenues des maisons particulières, tandis que quelques commerces et pubs se sont installés dans les rues principales.

62 Les rares menaces sont les modifications de fenêtres, portes, entourages, effectuées par des propriétaires avant le classement et surtout le passage, au cœur du village, d'une route à fort trafic.

63 L'élargissement de la notion de patrimoine industriel est non seulement spatial, du bâtiment seul à la ville entière, mais aussi temporel, avec la préservation d'éléments plus récents. C'est le cas de la cité Bata d'East Tilbury (East). La ville-usine, construite en 1933, est classée en conservation area depuis 1993. À l'intérieur, plusieurs bâtiments (des cités et l'ancien bâtiment de l'administration) sont classés grade II. East Tilbury se caractérise également par son environnement très rural malgré la proximité du Grand Londres et, comme à Saltaire, c'est l'homogénéité de l'ensemble qui fait sa richesse. Néanmoins, les menaces y sont plus fortes.

64 En 2009, l'ancienne usine, composée de trois gros bâtiments et de 10 autres plus petits, est en état de déprise manifeste depuis sa revente en 1998 ; les nouveaux propriétaires ont loué les bâtiments annexes à de petites entreprises, provoquant un mitage foncier, et les trois principaux bâtiments de l'usine sont vides ou presque. Enfin, les cités ont été revendues à des particuliers et ont donc connu des altérations inhérentes à leur personnalisation. Plusieurs équipements ont été détruits, comme la piscine, par exemple, et le but de la conservation area est à la fois de préserver l'ensemble de ce qui subsiste, d'entretenir et de conserver les arbres, les haies, le cadre originel de la villeusine et d'aider les propriétaires à redonner à leurs maisons, notamment en termes de fenêtres et d'entourage, leur caractère originel. Néanmoins, de lourdes interrogations subsistent sur le sort de l'usine et notamment sur celui des trois grands bâtiments qui se dégradent. De plus, la location des bâtiments plus petits à de nombreuses petites entreprises nuit à la cohérence de l'ensemble (www.thurrock.gov.uk).

Ces deux derniers exemples montrent donc que des villes-usines peuvent être préservées à titre patrimonial. Si elles sont évidement différentes par leur époque de construction et par leur statut de protection, elles ont en commun l'homogénéité préservée, la cohérence d'ensemble, qui en font des éléments exceptionnels au delà du simple intérêt qu'aurait pu représenter la conservation de l'usine seule. Cette cohérence peut aussi être sensible à une échelle encore plus petite que celle de la villeusine.

\section{B - La préservation de territoires industriels plus vastes}

Suivant le mouvement général d'élargissement de la notion de patrimoine, le patrimoine industriel concerne aujourd'hui des territoires de plus en plus vastes, à 
l'échelle des bassins ou des vallées industrielles. Il est en effet apparu que certains des territoires les plus largement transformés par l'industrie, tant en termes de durée (du $\mathrm{XVIII}^{\mathrm{e}}$ au XXe siècles au Royaume-Uni par exemple) que de taille (un quadrilatère de 116 sur $67 \mathrm{~km}$ pour la Ruhr, en Allemagne), recelaient tant de traces et d'héritages industriels que ces derniers devenaient incontournables dans l'optique d'une reconversion ou même d'une simple évolution.

Dans ces vastes territoires, bassins ou vallées industrielles, le patrimoine ne se résume pas à une seule usine entourée de cités ou d'annexes industrielles indirectes. Il s'agit plutôt d'un paysage entier créé par l'industrie, un cultural landscape, construit par l'activité humaine. Ce paysage est formé de la juxtaposition de très nombreuses villesusines ou villes-mines fonctionnant en concurrence et/ou en complémentarité. L'ensemble est à la fois un paysage issu des activités mais aussi le cadre de vie, durant plusieurs décennies voire plusieurs siècles, d'une population qui lui est attachée.

Complétant ce caractère identitaire évident, la profondeur chronologique et l'ampleur de la marque de l'industrie sur le territoire sont telles que le paysage entier, plus que l'usine seule, semble aujourd'hui constituer un témoignage de l'histoire de l'humanité, autant qu'un monument antique, qu'une vieille ville médiévale ou qu'un paysage rural préservé. À ce titre, on peut considérer que ce genre de paysage mérite d'être protégé et mis en valeur.

69 Si cet élargissement est d'abord sensible en Allemagne, avec la préservation combinée de nombreux sites de la vallée de l'Emscher, dès 1989, dans une Ruhr qui semble précurseur en la matière, les Britanniques ont rapidement suivi sur des territoires assez variés. Les meilleurs exemples de ce genre de larges territoire préservés sont les paysages miniers et industriels de Blaenavon au Pays-de-Galles, ou encore les paysages miniers des Cornouailles et du Devon, qui mettent en réseau plusieurs anciennes mines et usines liées au traitement du charbon ou de l'étain. Cette organisation réticulaire a d'ailleurs permis le classement de ces deux ensembles au patrimoine mondial de l'UNESCO.

70 Un autre exemple emblématique, également classé au patrimoine mondial, de cet élargissement des territoires du patrimoine industriel est la vallée de la Derwent (planche 3), dans les East Midlands. Cette vallée est considérée comme le lieu de naissance de l'industrie textile au XVIII ${ }^{e}$ siècle. Il y a d'abord, à Derby, la filature mécanique de la soie Lombe construite dans les années 1720 et considérée comme la première usine du monde, mais surtout, à Cromford, les premières filatures modernes, construites dans les années 1770 par Richard Arkwright. Les fabriques Arkwright sont imitées ensuite localement, mais aussi dans tout le Royaume-Uni (à New Lanark par exemple) et au delà (Allemagne, France, États-Unis).

71 Une grande proportion des usines de la Derwent existe toujours. À côté des usines, les éléments annexes subsistant sont aussi extrêmement nombreux, des infrastructures liées à la maîtrise de l'eau, essentielle comme source d'énergie à l'époque, aux constructions sociales des industriels, au premier rang desquelles les premières cités ouvrières. Les anciennes villes-usines de Cromford, Belper, Milford et Darley Abbey, etc. sont ainsi préservées et c'est en fait toute la vallée, le long de la rivière, élément à l'origine des investissements, qui, sur $24 \mathrm{~km}$, présente un paysage d'une grande cohérence. Il offre des caractéristiques évoquant la charnière des XVIII ${ }^{e}$ et XIX ${ }^{e}$ siècles et la naissance de la révolution industrielle, ce qui a motivé son classement. 
L'ensemble comporte 838 bâtiments inscrits sur la liste des bâtiments d'intérêt architectural ou historique particulier, dont 18 en grade I, essentiellement à Cromford. De plus, l'étendue de la zone inscrite au patrimoine mondial fait que des zones protégées à divers titres (parcs et jardins, sites de vie sauvage, préservation des habitats naturels, monuments, etc.) y sont incluses. Par ailleurs, la vallée reste un territoire vivant et un axe de circulation important à l'échelle régionale, ce qui soumet le territoire classé à des risques d'altération forts et en complique la gestion. Cette complexité est une des conséquences majeures de l'élargissement des territoires protégés.

Planche 3 : La Derwent Valley (East Midlands), une vallée industrielle textile entièrement classée au patrimoine mondiale de l'UNESCO

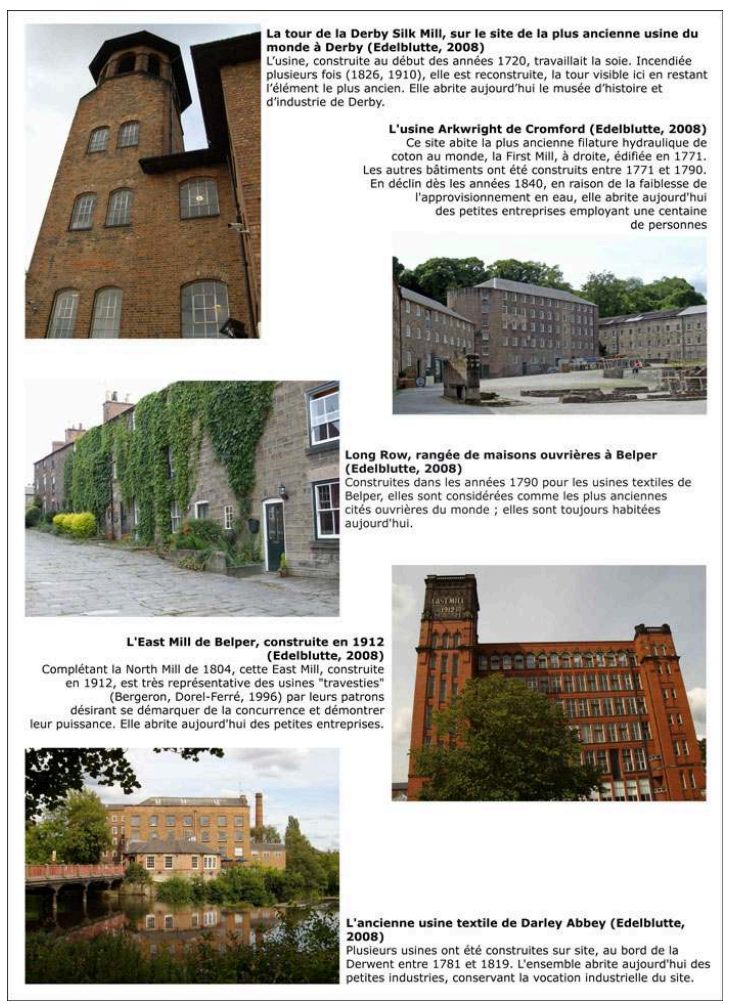

En effet, la variété des propriétaires est grande. L'essentiel des logements est privé, comme certaines anciennes usines, découpées en plusieurs lots occupés par des PME toujours actives. Certains grands bâtiments appartiennent cependant aux autorités locales, à l'État ou à des institutions caritatives. Le territoire inscrit, en raison de son étendue, dépend d'autorités locales diverses et emboîtées: districts de Derbyshire Dales, Amber Valley et Erewash, inclus dans le comté du Derbyshire et l'autorité unitaire $^{13}$ de Derby City. La gestion de l'ensemble est donc complexe.

Dans les années 1970, l'association Arkwright Society a attiré l'attention sur ce patrimoine exceptionnel. Ensuite, les autorités locales et English Heritage ont mis en place une stratégie d'urgence de protection et de mise en valeur, motivée par les forts risques d'individualisation des cités, revendues à des particuliers après l'effondrement des entreprises qui les géraient auparavant. Ensuite, le Derwent Valley Mills Steering Panel, organisme de coordination entre les différents acteurs (publics et privés), est mis en place en 1997, sous la direction effective du Conseil du Comté du Derbyshire. Il travaille dans des domaines variés, identifiant les risques encourus pour les territoires 
classés, assurant le lien entre les propriétaires privés et les autorités locales, proposant des voies de développement et d'aménagement, notamment routiers (whc.unesco.org). De nombreux exemples de partenariats complexes entre secteurs public et initiatives privées ont été ainsi mis au point dans tout le Royaume-Uni pour protéger ces vastes territoires. Des exemples détaillés de ces montages financiers sont développés dans l'ouvrage édité par M. Stratton, Industrial buildings, conservation and regeneration (2000). La comparaison entre la taille du territoire de la Derwent (allongé sur 24km), inscrit au patrimoine mondial en 2001 et celui d'Ironbridge (allongé sur 4km), inscrit en 1986, illustre finalement l'élargissement récent apporté à la notion de patrimoine industriel (figure 4).

Figure 4 : La Derwent Valley (East Midlands) et l'Ironbridge Gorge (West Midlands), superficies comparées des zones inscrites au patrimoine mondial de l'UNESCO

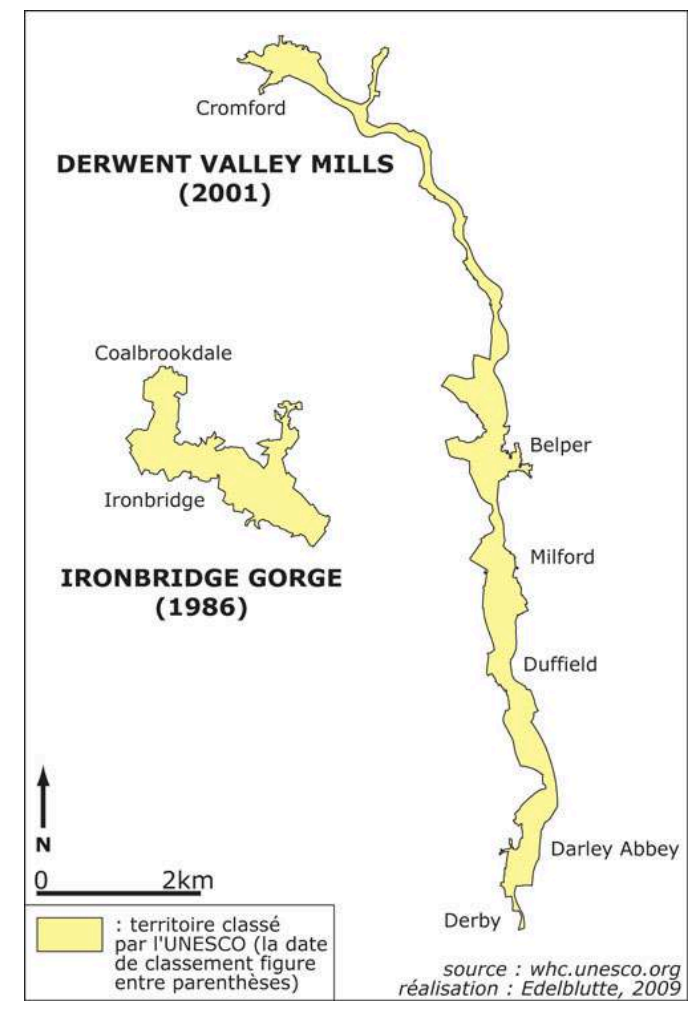

\section{C - La mise en réseau pratique du patrimoine industriel}

La mise en réseau de sites proches permet la protection de territoires plus vastes. Mais elle s'opère aussi à l'échelle continentale et mondiale, dans le cadre d'organismes mis en place récemment. Le Royaume-Uni y tient une place essentielle, même s'il n'a pas été à l'origine du principal organisme européen.

Fédérant sites et territoires industriels à l'échelle du continent, la Route Européenne du Patrimoine industriel (European Route of Industrial Heritage - ERIH), calquée sur le modèle de la Route der Industriekultur qui relie les principaux anciens sites industriels de la Ruhr, est mise en place à partir de 2002, à l'initiative du Land de Rhénanie NordWestphalie et dans le cadre d'un programme INTERREG II C. 
Il s'agit d'un réseau de lieux industriels, composé d'abord de points d'ancrage, c'est-àdire de lieux phares et symboles du patrimoine industriel, dans lequel on retrouve, entre autres, pratiquement tous les sites majeurs évoqués plus haut. En 2009, des sites de 7 pays participent à ce réseau. L'essentiel se trouve en Allemagne, au Royaume-Uni et aux Pays-Bas, les trois premiers pays partenaires, mais d'autres ont intégré le réseau, en Belgique, au Luxembourg, en République tchèque et en France (www.erih.net). Par ailleurs, l'ERIH propose :

- des itinéraires régionaux, ancrés sur les lieux forts évoquées ci-dessus mais reliant en plus d'autres sites moins emblématiques;

- des itinéraires thématiques (textile, mine, sidérurgie, énergie, eau, transports, etc.) qui intègrent, hors itinéraires régionaux, plusieurs centaines de sites dans toute l'UE, en Suisse, en Norvège et en Turquie ;

- des informations très complètes sur l'histoire industrielle et sur chaque ancien site industriel et minier référencé.

Figure 5 : Points d'ancrage, sites et itinéraires régionaux de la Route Européenne du Patrimoine Industriel en 2009 au Royaume-Uni

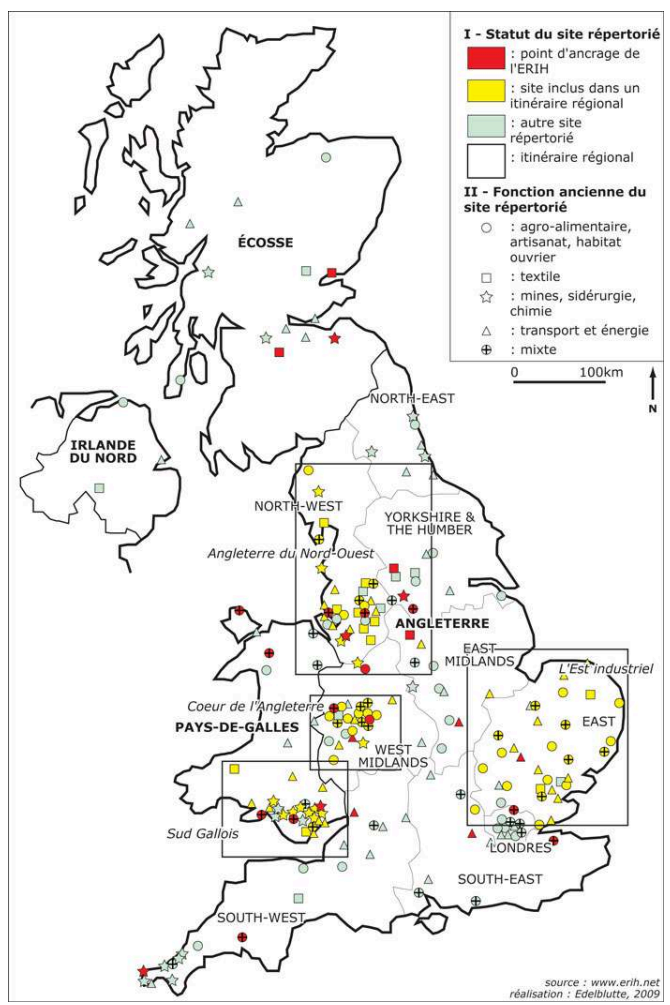

La figure 5 présente les sites britanniques recensés par l'ERIH, le Royaume-Uni étant le pays qui en compte le plus (cf. supra). Cependant, le recensement a été réalisé de façon large en incluant des éléments ne pouvant être que marginalement rattachés à l'industrie; de plus, il ne s'est opéré qu'en accord avec les sites recensés, ce qui explique l'absence de certains, parfois très importants (le Beamish Open Air Museum, par exemple) et la présence de sites nombreux quand il y a la volonté d'intégrer un itinéraire régional.

80 Ainsi, une région comme l'East, historiquement peu industrialisée, ressort-elle plus sur la carte que le North-East ou les Lowlands d'Écosse, pourtant espaces très précocement et très profondément industrialisés. La carte traduit certes une réelle densité de sites 
industriels patrimoniaux (North-West, Sud du Pays-de-Galles, West Midlands), mais plus encore l'intensité plus ou moins affirmée, aux niveaux local et régional, de la volonté de préservation et de mise en valeur du patrimoine industriel.

81 Enfin, ces sites recensés ne représentent cependant qu'une partie de ce que l'on peut classer patrimoine industriel car, si certains sites reconvertis vers le commerce ou la culture apparaissent, ce n'est pas le cas de ceux devenus résidences, bureaux ou nouvelles industries...

Malgré les imperfections de ce recensement, l'ERIH est devenue, en plus d'un réseau reliant les sites du patrimoine industriel, une banque d'informations extraordinaire et incontournable dans ce domaine en Europe, consacrant la notion de patrimoine industriel à l'échelle du continent presque tout entier.

Au niveau mondial, les associations nationales ont vite vu l'intérêt de se fédérer. Cela s'est effectué par l'intermédiaire du TICCIH (The International Committee for the Conservation of Industrial Heritage - Conseil international pour la préservation du patrimoine industriel), fondé en 1973 lors d'un congrès à Ironbridge ${ }^{14}$. Il organise tous les 3 ans d'importantes rencontres internationales, rassemblant plusieurs centaines de personnes, sur des hauts lieux du patrimoine industriel mondial.

Le TICCIH adopte en juillet 2003, à Nijni-Taguil, une des plus anciennes villes-usines de l'Oural (Russie), une charte définissant le patrimoine industriel et ses méthodes. Le préambule précise que "le patrimoine industriel comprend les vestiges de la culture industrielle qui sont de valeur historique, sociale, architecturale ou scientifique. Ces vestiges englobent: des bâtiments et des machines, des ateliers, des moulins et des usines, des mines et des sites de traitement et de raffinage, des entrepôts et des magasins, des centres de production, de transmission et d'utilisation de l'énergie, des structures et infrastructures de transport aussi bien que des lieux utilisés pour des activités sociales en rapport avec l'industrie (habitations, lieux de culte ou d'éducation)» (www.mnactec.cat/ticcih). La conception du patrimoine industriel est donc bien plus large que celle pratiquée au début du mouvement.

Plus précisément, la charte définit ensuite l'archéologie industrielle comme l'ensemble des méthodes utilisées, dans un cadre interdisciplinaire, pour l'étude du patrimoine industriel. Enfin, la charte délimite chronologiquement la période d'étude, du milieu du XVIII ${ }^{e}$ siècle à nos jours, "sans négliger ses racines pré et proto-industrielles" (www.mnactec.cat/ticcih).

86 Par ailleurs, le TICCIH publie depuis 1999 une revue «Patrimoine de l'industrie / Industrial Patrimony " (www.koinetwork.org) et est aussi le conseiller en matière de patrimoine industriel pour ICOMOS (The International Council on Monuments and Sites Conseil international des monuments et des sites), organisme non gouvernemental fondé en 1965 et conseillant l'UNESCO (United Nations Educational, Scientific and Cultural Organization - Organisation des Nations Unies pour l'Éducation, la Science et la Culture) pour le classement des sites au patrimoine mondial.

87 Le patrimoine industriel britannique occupe justement une place de choix dans la liste du patrimoine mondial de l'UNESCO. Même si ce classement, comme celui de l'ERIH, n'est ni exhaustif, ni toujours exempt de motivations politiques plus que culturelles, il représente un critère essentiel pour les sites classés.

39 sites $^{15}$ concernant directement ou indirectement le patrimoine industriel sont inscrits sur cette liste et donc reconnus au niveau mondial. Le premier a été les mines de sel de Wieliczka, en Pologne, protégé à ce niveau dès 1978 ; le second, l'Ironbridge 
Gorge Park, inscrit en 1986. Certes, ces 39 sites ne représentent que 4,4\% des 878 sites inscrits en février 2009, mais ils sont majoritairement localisés en Europe (29 sur 39) et plus particulièrement en Europe nord-occidentale. Sept sont au Royaume-Uni, ce qui confirme encore l'avance et la primauté de ce pays en la matière (figure 6).

Figure 6 : Sites industriels ou partiellement industriels du patrimoine mondial de l'UNESCO en Europe

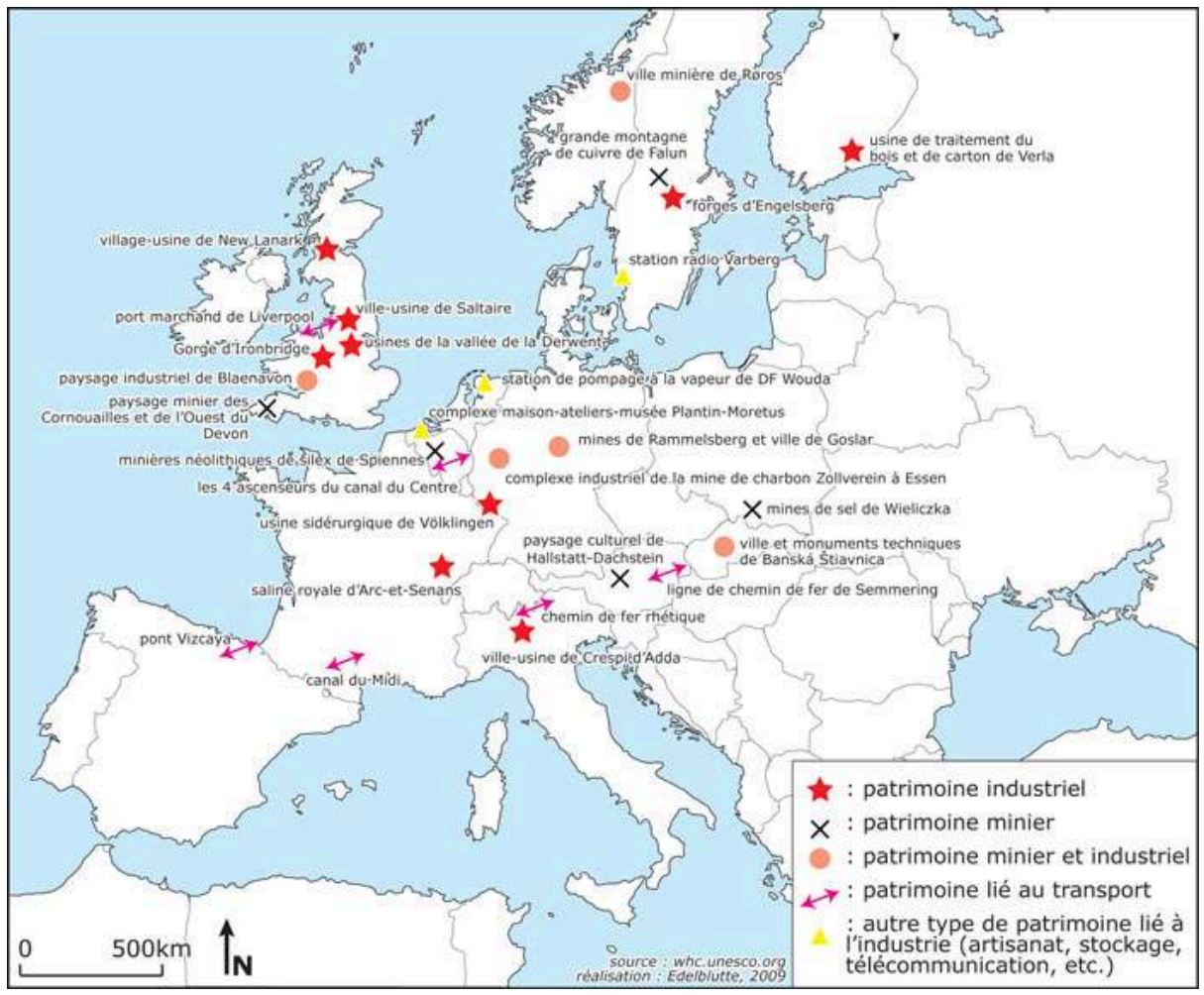

89 Ces 7 sites industriels représentent un peu plus d'un quart des sites britanniques inscrits sur la liste du patrimoine mondial. Ils l'ont néanmoins été, pour la majorité, très récemment et justement en lien avec l'élargissement de la notion de patrimoine industriel et la mise en réseau de sites industriels proches. Il est en effet difficile de classer une seule usine ${ }^{16}$, rarement considérée comme assez emblématique au niveau mondial (et, au delà, rarement assez attractive) alors qu'un ensemble de sites et villeusines possède un poids et une valeur plus fortes.

Ainsi, le premier site classé est celui de l'Ironbridge dès 1986 (il s'agit d'ailleurs, et très précocement, d'une mise en réseau de plusieurs musées et parcs, autour du célèbre pont), mais il faut attendre ensuite le $\mathrm{XXI}^{\mathrm{e}}$ siècle pour voir classer les vastes ensembles que sont Blaenavon (2000), la Derwent Valley, Saltaire, New Lanark (2001) ou encore le paysage minier des Cornouailles et du Devon (2006). Blaenavon et les Cornouailles sont d'ailleurs classés par l'UNESCO comme "paysages» intégrant donc cet ultime élargissement de la notion de patrimoine dans leur dénomination même.

\section{Conclusion}

Le Royaume-Uni, précocement industrialisé et précocement désindustrialisé suite aux crises du dernier quart du $\mathrm{XX}^{\mathrm{e}}$ siècle, est donc aussi le premier pays à avoir intégré la 
composante patrimoniale dans la reconversion des sites, puis des territoires industriels de plus en plus vastes, du quartier à la vallée et au bassin en passant par la ville-usine et la ville industrielle.

Cette place essentielle du patrimoine industriel, parfois un peu redondante avec par exemple les nombreuses mines-musées, est encore renforcée, dans la partie Nord de l'Angleterre autant qu'en Écosse et au Pays-de-Galles, par le fossé à la fois réel et fantasmé, existant entre ces régions et le grand Sud-Est de l'Angleterre autour de Londres.

La préservation des héritages industriels entre ainsi dans la préservation d'une identité qui se construit en opposition au grand Sud-Est. Cette identité intègre l'ancienne industrie que l'on a dit sacrifiée par les intérêts londoniens sous les gouvernements conservateurs de M. Thatcher et J. Major, sans d'ailleurs que le New Labour de T. Blair inverse ou même rectifie le mouvement lors de son arrivée au pouvoir en 1997. L'ancienne culture industrielle est même souvent au cœur de mouvements politiques nationalistes en Écosse et au Pays-de-Galles, ou régionalistes dans les régions du Nord de l'Angleterre (Bailoni, 2007).

$94 \mathrm{Au}$ delà des préservations patrimoniales et des motivations politiques, l'intégration du mythe industriel à la culture populaire est une constante dans le Nord britannique, au moins depuis les années 1980 et la crise. L'esthétique industrielle de certaines œuvres d'art (la statue Angel of the North à Gateshead, dans le Nord-Est), la multiplication de films sociaux et à succès ayant pour cadre l'Angleterre industrielle en crise des années 1980 (" The full monty - Le grand jeu », de Peter Cattaneo, "Brassed off - Les virtuoses », de Mark Herman, ou encore «Billy Elliot », de Stephen Daldry), les concerts donnés dans d'anciennes usines ou raffineries en sont autant d'exemples, comme la réalisation de pièces artistiques originales sur le thème industriel.

Ainsi, le groupe pop-rock liverpuldien Orchestral Manoeuvres in the Dark, le vidéaste Hambi Haralambus et le designer Peter Saville ont-ils créé une œuvre à la fois sonore et visuelle (The Energy Suite), combinant images et sons de 5 sites industriels (dont une centrale nucléaire) du Nord-Ouest et présentée à la Foundation for Art and Creative Technology de Liverpool de décembre 2008 à février 2009. Cette œuvre, par ailleurs jouée en juin 2009 en compagnie du Royal Liverpool Philarmonic Orchestra (www.culture24.org.uk et www.omd.co.uk), illustre l'intégration pleine à l'identité locale de cette culture industrielle qui s'étend donc largement au delà du simple souvenir d'une époque glorieuse mais révolue.

\section{BIBLIOGRAPHIE}

Andrieux J.-Y. (1992). - Le patrimoine industriel, Que sais-je ?, n²657, Paris, Presses Universitaires de France, $127 \mathrm{p}$.

Bailoni м. (2007). - La question régionale en Angleterre. Nouvelles approches politiques du territoire anglais, Thèse de Doctorat, Université Paris 8,560 p. 
Bailoni M. (2009). - « Quelle place pour le patrimoine dans le renouveau d'une région postindustrielle? Le cas du Nord-Est anglais ", Revue Géographique de l'Est, 1-2/2008, www.revues.org.

Belhoste J.-F., Smith P. - Dir. (1997). - Patrimoine industriel, 50 sites en France, Paris, Éditions du patrimoine, $128 \mathrm{p}$.

Bergeron L., Dorel-Ferré G. (1996). - Le patrimoine industriel, un nouveau territoire, Paris, Liris, $127 \mathrm{p}$.

Buchanan R. A. (1972). -Industrial Archaeology in Britain, Harmondsworth, Pelican, 444 p.

Burgel G. (2001). - « Mémoire de la ville et recomposition urbaine ». In : Loyer F. - dir., Ville d'hier, ville d'aujourd'hui, Paris, Fayard - Éditions du Patrimoine, p. 95-104.

Cartier C. (2003). - L'héritage industriel, un patrimoine, Besançon, CRDP de Franche-Comté, 195 p. Casella E., Symonds J. (2005). - Industrial archeology, future directions, New York, Springer, 321 p. Chaline C. (1999). - La régénération urbaine, Paris, Presses Universitaires de France, coll. Que saisje ?, $127 \mathrm{p}$.

Chassagne S. (2002). - « L'élargissement d'un concept : de l'archéologie (industrielle) au patrimoine (industriel) », Le Mouvement Social, n²00, p. 7-9.

Choay F., Merlin P. (2005). - Dictionnaire de l'urbanisme et de l'aménagement, Paris, Presses Universitaires de France, 964 p.

Cossons N. (1975). - The BP book of industrial archeology, Newton Abbot, David \& Charles, $496 \mathrm{p}$.

Cossons N. - éd. (2000). - Perspectives on industrial archeology, Londres, Science Museum, 176 p.

Cullingworth B., Nadin V. (2006). - Town and country planning in the UK, 14ème edition, Londres, Routledge, $587 \mathrm{p}$.

Daumas M. (1980). - L'archéologie industrielle en France, Paris, Robert Laffont, 463 p.

De Roux E. (2000). - Patrimoine industriel, Éditions du patrimoine et éditions Scala, 271 p.

Deshaies M. (2007). - Les territoires miniers. Exploitation et reconquête, Paris, Ellipses, 224 p.

Edelblutte S. (2009). - « Quesont devenues les villes-usines ? Réflexion à partir du cas lorrain ». In : Vallat C. - Dir., Pérennité urbaine ou la ville par delà ses métamorphoses. Volume 3 - Essence, Paris, L'Harmattan, p. 137-153.

Edelblutte S. (2008). - « Que reste-t-il du textile vosgien? », L'Information géographique, n²/72, p. 66-88.

Edelblutte S. (à paraître fin 2009). - Paysages et territoires de l'industrie en Europe : héritages et renouveau, Paris, Ellipses.

Falconer K. (1980). - Guide to England's Industrial Heritage, Londres, Batsford, 270 p.

Falconer K. (2006). - « The industrial heriatge in Britain - the first fifty years ", La revue pour l'histoire du CNRS, n¹4, 11 p., http://histoire-cnrs.revues.org/document1778.html, 5 juillet 2009.

Hudson K. (1963). - Industrial archeology: an introduction, Londres, J. Baker, 179 p.

Hudson K. (1971). - A Guide to the Industrial Archaeology of Europe, Bath, Adams\& Dart, 186 p. Leboutte R. (1997). - Vie et mort des bassins industriels en Europe ; 1750-2000, Paris, L'Harmattan, $591 \mathrm{p}$. 
Lévy J., Lussault M. (2003). - Dictionnaire de la géographie et de l'espace des sociétés, Paris, Belin, $1033 \mathrm{p}$.

Nenadic S. (2002). - « Historiens et patrimoine en Grande-Bretagne », Le Mouvement Social, $\mathrm{n}^{\circ} 200$, p. 116-122.

Palmer M., Neaverson P. (1998). - Industrial archeology, principles and practice, Londres, Routledge, $180 \mathrm{p}$.

Stratton M. (2000). - Industrial buildings, conservation and regeneration, Londres, Spon, 256 p.

Veschambre V. (2005). - « Le recyclage urbain, entre démolition et patrimonialisation : enjeux d'appropriation symbolique de l'espace. Réflexions à partir de quatre villes de l'Ouest ", Norois, $\mathrm{n}^{\circ} 2$, p. 79-92.

\section{SITES INTERNET CONSULTÉS ENTRE JUILLET 2008 ET JUIN 2009 :}

www.unesco.org : site du patrimoine mondial de l'UNESCO

www.culture24.org.uk : site de promotion de la culture au Royaume-Uni

www.erih.net : site de la Route Européenne du Patrimoine Industriel - European Route of Industrial Heritage, réseau de sites du patrimoine industriel européen

www.erih.net : site de la Route Européenne du Patrimoine Industriel - European Route of Industrial Heritage, réseau de sites du patrimoine industriel européen

www.industrial-archaeology.org.uk : site de The Association for Industrial Archaeology, organisme britannique consacré à la préservation du patrimoine industriel national

www.ironbridge.org.uk : site officiel de l'Ironbridge Gorge Park

www.koinetwork.org : site d'un Groupement européen d'intérêt économique, agence pour l'internationalisation des cultures et des entreprises, créée en 2002

www.mnactec.cat/ticcih : site officiel du TICCIH, The International Committee for the Conservation of Industrial Heritage - Conseil international pour la préservation du patrimoine industriel

www.omd.co.uk : site officiel du groupe pop-rock Orchestral Manoeuvres in the Dark

www.tourismtrade.org.uk : site de VisitBritain, organisme officiel de promotion du tourisme au Royaume-Uni

\section{NOTES}

1. «Pays de Tradition industrielle» (PTI) est préféré ici à «Pays Anciennement industriel» ou «Post-industriel», expressions qui impliquent une disparition de l'industrie, tant dans sa forme active que dans sa forme patrimoniale. L'expression peut être utilisée à plus grande échelle à propos des régions (RTI).

2. Ce terme désigne des formes de travail préindustrielles concentrant plusieurs centaines d'ouvriers (manufactures royales en France), mais sans mécanisation, ou encore des formes légèrement mécanisées mais encore très artisanales et avec des effectifs faibles (moulins, tisserands à domicile). La proto-industrie prend place avant la première révolution industrielle débutant au XVIII ${ }^{\mathrm{e}}$ siècle au Royaume-Uni.

3. La ville-usine est née de l'industrie; elle a été totalement ou presque totalement créée par l'industriel autour de la mine ou de l'usine. Elle peut être distingué de la ville industrielle, ville déjà existante accueillant des usines (Edelblutte, 2008, 2009). 
4. English Heritage est spécifique à l'Angleterre. Ses équivalents sont Historic Scotland en Écosse, Cadw au Pays-de-Galles et Environment and Heritage Service en Irlande du Nord.

5. National Trust est une association à but non lucratif, dont la création en 1895 était justement inscrite en réaction face à l'urbanisation et l'industrialisation croissante du Royaume-Uni. C'est donc d'abord à la protection du patrimoine rural et du patrimoine naturel qu'elle s'est attachée, dominée par la nostalgie d'une Angleterre verte en voie de disparition. Elle est devenue depuis lors le second propriétaire privé du pays après la Couronne et ce n'est qu'assez récemment qu'elle intègre des éléments urbains et donc aussi des bâtiments industriels. National Trust est compétent en Angleterre, au Pays-de-Galles et en Irlande du Nord. En Écosse, c'est National Trust for Scotland qui opère.

6. L'exemple des Docks de Liverpool, transformés assez rapidement en vaste centre commercial autour du Tate Liverpool Museum (musée d'art moderne et contemporain, antenne du Tate Modern de Londres) est emblématique de ce genre de politique.

7. Les territoires de référence sont ceux des 3 Nations périphériques du Royaume-Uni et des régions en Angleterre, toutes délimitées sur la figure 4.

8. Les usines tubulaires désignent les usines sidérurgiques et chimiques, où les imposantes machines, tels que les hauts-fourneaux, les tours de distillation, etc. sont apparentes et entourées de bandes transporteuses et de conduites multiples, d'où leur nom.

9. Voir l'article de M. Bailoni, dans ce numéro, à propos des musées nés autour des mines de plomb de Killhope (North-East) et de Nenthead (North-West), distants de quelques kilomètres, mais dans deux comtés et régions différentes.

10. Les sheds, connus aussi en France sous le nom de "toits en dents de scie ", sont des toitures décomposées en plusieurs pans. Les pans orientés au Sud sont couverts de tuile et ceux orientés au Nord sont vitrés, autorisant, pour des bâtiments de plain pied, vastes et aveugles, un éclairage indirect efficace et non gênant pour le travail du textile.

11. Le propriétaire actuel, Boyd Hargreaves, est le descendant de James Hargreaves, l'inventeur de la Spinning Jenny, machine à tisser emblématique et pilier de la révolution industrielle dans le textile.

12. Le grade I désigne des édifices "d'un intérêt exceptionnel ", le grade II* des édifices "particulièrement importants et d'un intérêt spécial » et le grade II « des édifices d'un intérêt spécial ». Cela implique essentiellement que le bâtiment classé ne peut pas être démoli, agrandi ou altéré sans une permission spéciale des autorités locales compétentes en matière d'urbanisme. 13. Système de gouvernement local à niveau unique, qui se distingue du système à double niveau comté/district.

14. Les trois premiers congrès (Ironbridge, 1973, Bochum, 1976 et Stockholm, 1978) ont été organisés sous le nom de Conférence Internationale pour la Conservation des Monuments (et non du Patrimoine) Industriels, soulignant encore l'élargissement progressif de la notion.

15. Le décompte est large et inclut des sites industriels, miniers, miniers et industriels et de transport (notamment les voies ferrées et les canaux). Seuls 17 sites sont purement industriels ou miniers et industriels.

16. C'est néanmoins le cas en Allemagne avec l'ancienne usine sidérurgique de Völklingen (Sarre), ou encore en France avec la saline proto-industrielle d'Arc-et-Senans (Franche-Comté). 


\section{RÉSUMÉS}

Le patrimoine industriel est l'un des derniers types de patrimoine à être protégé. Cependant, le mouvement de protection est particulièrement précoce au Royaume-Uni, principalement en raison de la profondeur de l'industrialisation de ce pays qui a vu naître l'industrie.

Cet article évoque l'image et la diversité de ce patrimoine à travers l'étude de ses paysages et de ses territoires, de la grande à la petite échelle.

Il débute par une brève analyse du mouvement, replacé dans un contexte international. Il montre ensuite plusieurs exemples de conservation et/ou de préservation, d'une ruine maintenue en l'état à une zone commerciale réutilisant un ancien site industriel et ses usines. Il insiste ensuite sur l'élargissement des territoires protégés en tant que patrimoine industriel, d'un seul objet industriel placé dans un square pour rappeler l'ancienne gloire industrielle d'une ville, à une vallée entière ou un bassin charbonnier de plusieurs centaines de $\mathrm{km}^{2}$, incluant, par exemple, plusieurs villes-usines ou mines. Enfin, cet article traite de la mise en réseau croissante du patrimoine industriel britannique, à différents niveaux : européen et mondial, avec l'inscription d'éléments industriels au patrimoine mondial de l'UNESCO.

Industrial heritage is one of the last types of heritage being protected. However, the movement of protection is especially early in the United Kingdom. This precocity is largely due to the depth of the industrialization in the birth country of industry.

This paper deals with the image and the diversity of this heritage through its landscapes and territories, from the large scale to the small scale.

It deals first with a brief analysis of this movement, seen in an international context. Secondly, it shows several examples of conservation and/or preservation, from a ruin maintained in an unchanged state to a commercial estate reusing an old industrial site and its factories. It insists on the widening of the territories protected as industrial heritage, from a single industrial object placed on a square to remind the previous industrial glory of a city, to an entire valley or a coal basin of several hundred $\mathrm{km}^{2}$, including, for example, several company-towns or mines. At last, this paper deals with the growing networks around British industrial heritage at several levels: European level or World level with the inscription of industrial elements on UNESCO's World Heritage Sites.

Das industrielle Erbe ist eine der letzten, die unter Schutz gestellt wurde. Im Vereinigten Königreich setzt jedoch die Schutzbewegung besonders früh ein, insbesondere wegen des starken Gepräges der Industrialisierung im Land, der Keimzelle der Industrialisierung.

Durch eine Untersuchung der Landschaften und der Räume von der lokalen bis zur regionalen Maßstabebene erwähnt dieser Artikel das Bild und die Vielfalt dieses Erbes. Er beginnt mit einer kurzen Analyse der Schutzbewegung in einem internationalem Rahmen. Er zeigt mehrere Beispiele von der Erhaltung einer industriellen Ruine bis zu einem Einzelhandelstandort, der auf einem ehemaligen industriellen Standort entstanden ist. Er legt dann den Akzent auf die Erweiterung der Schutzgebiete für das industrielle Erbe, von einem industriellen Denkmal auf einer kleinen Grünanlage als Erinnerung des Glanzes vergangener industriellen Zeit, bis zu einer Stadt, einem ganzen Tal oder einem ganzen Kohlenbergbaurevier mehrerer hundert km2 mit z. B. mehreren Fabrikstädten oder Bergwerken. Schließlich erwähnt dieser Artikel die Netzaufstellung des britannischen industriellen Erbes auf unterschiedlichen Niveaus : europäisch und weltweit mit der Antragung von industriellen Bestandteilen auf die Welterbeliste. 
INDEX

Schlüsselwörter : Industrie, industrielles Erbe, Landschaft, Umgestaltung, Vereinigtes Königreich

Keywords : industrial heritage landscape, industry, regeneration, United-Kingdom Mots-clés : industrie, patrimoine industriel, paysage, reconversion, Royaume-Uni

\section{AUTEUR}

\section{SIMON EDELBLUTTE}

Département de Géographie, Centre d'Études et de Recherches sur les Paysages (CERPA), Université Nancy2 - BP 33-97 - 54015 NANCY Cedex - simon.edelblutte@univ-nancy2.fr 